\title{
PAHSAs enhance hepatic and systemic insulin sensitivity through direct and indirect mechanisms
}

\author{
Peng Zhou, ${ }^{1}$ Anna Santoro, ${ }^{1}$ Odile D. Peroni, ${ }^{1}$ Andrew T. Nelson, ${ }^{2}$ Alan Saghatelian, ${ }^{3}$ Dionicio Siegel, ${ }^{2}$ and Barbara B. Kahn \\ 'Division of Endocrinology, Diabetes and Metabolism, Department of Medicine, Beth Israel Deaconess Medical Center and Harvard Medical School, Boston, Massachusetts, USA. \\ ${ }^{2}$ Skaggs School of Pharmacy and Pharmaceutical Sciences, UCSD, La Jolla, California, USA. ${ }^{3}$ Clayton Foundation Laboratories for Peptide Biology, Helmsley Center for Cenomic Medicine, \\ Salk Institute for Biological Studies, La Jolla, California, USA.
}

\begin{abstract}
Palmitic acid esters of hydroxy stearic acids (PAHSAs) are bioactive lipids with antiinflammatory and antidiabetic effects PAHSAs reduce ambient glycemia and improve glucose tolerance and insulin sensitivity in insulin-resistant aged chowand high-fat diet-fed (HFD-fed) mice. Here, we aimed to determine the mechanisms by which PAHSAs improve insulin sensitivity. Both acute and chronic PAHSA treatment enhanced the action of insulin to suppress endogenous glucose production (ECP) in chow- and HFD-fed mice. Moreover, chronic PAHSA treatment augmented insulin-stimulated glucose uptake in glycolytic muscle and heart in HFD-fed mice. The mechanisms by which PAHSAs enhanced hepatic insulin sensitivity included direct and indirect actions involving intertissue communication between adipose tissue and liver. PAHSAs inhibited lipolysis directly in WAT explants and enhanced the action of insulin to suppress lipolysis during the clamp in vivo. Preventing the reduction of free fatty acids during the clamp with Intralipid infusion reduced PAHSAs' effects on EGP in HFD-fed mice but not in chow-fed mice. Direct hepatic actions of PAHSAs may also be important, as PAHSAs inhibited basal and glucagon-stimulated EGP directly in isolated hepatocytes through a cAMP-dependent pathway involving $\mathrm{C} \alpha_{\mathrm{i}}$ protein-coupled receptors. Thus, this study advances our understanding of PAHSA biology and the physiologic mechanisms by which PAHSAs exert beneficial metabolic effects.
\end{abstract}

\section{Introduction}

Type 2 diabetes (T2D) is a worldwide epidemic that affected 425 million people in 2017, as estimated by the International Diabetes Federation (1). The major pathogenic factors in T2D are resistance to the actions of insulin in adipose tissue, muscle, and liver, and dysregulated insulin secretion. Whole-body glucose homeostasis is maintained primarily by a balance between endogenous glucose production (EGP), mainly from the liver, and glucose uptake by muscle, adipose tissue, and liver (2). EGP is regulated by hormones, substrates, and neuronal signals. For example, glucagon and catecholamines activate $\mathrm{G} \alpha$ s protein-coupled receptors and increase EGP through a cAMP-dependent pathway, which involves PKA activation (3). On the other hand, insulin suppresses EGP directly through an insulin receptor/PI3K pathway and indirectly through the suppression of adipose tissue lipolysis (4-7). Impaired suppression of EGP by insulin (direct or indirect) contributes to the fasting hyperglycemia in T2D. Hence, restoring hepatic and systemic insulin sensitivity is an effective approach to prevent and treat T2D. However, few insulin-sensitizing medications are currently available, and those that are available have

Authorship note: PZ and A. Santoro are co-first authors.

Conflict of interest: BBK and $A$. Saghatelian are inventors on patents related to PAHSAs: W02013166431A1 and W02017070515A3.

Copyright: (5) 2019, American Society for Clinical Investigation.

Submitted: December 28, 2018; Accepted: June 24, 2019; Published: August 26, 2019.

Reference information: J Clin Invest. 2019;129(10):4138-4150.

https://doi.org/10.1172/JCl127092. significant side effects and are not used widely $(8,9)$. Because hyperinsulinemia and insulin resistance have been implicated in the risk for cardiovascular disease, cancer, neurodegenerative diseases, and cognitive dysfunction (10-12), there is a major need for insulin sensitizers to reduce the burden of metabolic disease.

Our laboratory discovered a class of structurally novel bioactive lipids, branched fatty acid esters of hydroxy fatty acids (FAHFAs), that are synthesized in humans, animals, and plants, and have antidiabetic and antiinflammatory properties (13-15). FAHFAs consist of a fatty acid and a hydroxy fatty acid linked by an ester bond. An in silico analysis predicted that more than 1000 structurally distinct FAHFAs could exist (16), and we and others identified more than 20 FAHFA families in mammalian tissues and 49 families in plants (13-15). Serum levels of one FAHFA family, palmitic acid esters of hydroxy stearic acid (PAHSAs), correlate highly with insulin sensitivity in humans, and PAHSA levels in serum and subcutaneous (SQ) adipose tissue are lower in insulin-resistant compared with insulin-sensitive people (13). Furthermore, others showed that levels of 5-PAHSA in breast milk are negatively correlated with obesity in nursing mothers (17). PAHSA levels are also decreased in liver and SQ adipose tissue (but not in perigonadal adipose tissue) of mice with high-fat diet-induced (HFD-induced) insulin resistance (13). PAHSAs enhance glucose tolerance and insulin sensitivity in chow- and HFD-fed mice without altering adiposity $(13,18,19)$, potentiate insulin and GLP-1 secretion in aged chow-fed mice (18), and have antiinflammatory properties $(13,14,18,20)$. PAHSAs also augment glucose-stimulated insulin secretion in human islets from nondiabetic individuals and those with T2D $(13,18,21)$. Recent data 
also show that PAHSAs promote adipocyte differentiation (22) and browning (23). However, recently Pflimlin et al. (24) failed to find beneficial metabolic effects with a single dose or 6-day treatment with PAHSAs in HFD-fed mice (24). Many important methodological issues contributed to their negative results, and these are discussed extensively in recent publications $(25,26)$. In addition, another group using the same vehicle as Yore et al. (13) also found improved glucose tolerance in HFD-fed but not $d b / d b$ mice (27).

In the current study, we demonstrate using a more sophisticated technique, the hyperinsulinemic-euglycemic clamp, that PAHSAs are indeed insulin sensitizers. Furthermore, we identify additional targets of PAHSA action. PAHSA treatment enhances hepatic insulin sensitivity in aged chow- and HFD-fed mice and promotes glucose uptake into glycolytic muscle and heart in HFD-fed mice. To determine the mechanisms by which PAHSAs enhance hepatic insulin sensitivity, we examined both direct effects in hepatocytes and the indirect effect resulting from the augmentation of insulin-mediated suppression of white adipose tissue (WAT) lipolysis. Increased WAT lipolysis promotes hepatic insulin resistance by increasing hepatic gluconeogenesis through a substrate push mechanism (5). To determine whether lowering free fatty acid (FFA) levels is necessary for the effects of PAHSAs on insulin sensitivity, we infused Intralipid during the hyperinsulinemic-euglycemic clamp. PAHSAs still lowered EGP in chow-fed mice infused with Intralipid, whereas in HFD-fed mice, preventing the PAHSA-induced reduction in FFAs diminished the effects of PAHSAs on hepatic insulin sensitivity. Furthermore, our mechanistic studies show that PAHSAs inhibited EGP directly in isolated hepatocytes. This appears to be mediated by a cAMP-dependent pathway involving $\mathrm{G} \alpha_{\mathrm{i}}$ protein-coupled receptors. In summary, PAHSAs have direct and indirect effects on EGP, and these contribute to their systemic insulin-sensitizing effects. The indirect effects result from intertissue communication between adipose tissue and liver. Therefore, these data reveal what we think are new cellular and physiologic mechanisms underlying the beneficial effects of PAHSAs.

\section{Results}

Acute PAHSA treatment decreases glucose production in vivo through inhibition of cAMP signaling. A single oral dose of 5- and 9-PAHSAs, compared with vehicle treatment, decreased glycemia during a pyruvate tolerance test (PTT) in 18-hour-fasted chow-fed mice without changing insulin levels (Supplemental Figure 1; supplemental material available online with this article; https://doi. org/10.1172/JCI127092DS1). Consistent with this, acute 9-PAHSA infusion reduced EGP in 5-hour-fasted mice on a chow or HFD (Figure 1A). To determine whether reduced EGP with acute 9-PAHSA infusion leads to reduced glycemia, we measured glycemia at the end of the PAHSA infusion in chow- and HFD-fed mice. Acute 9-PAHSA infusion tended to reduce glycemia in mice on a chow diet (Figure 1B, left), consistent with reduced EGP. However, 9-PAHSA did not change ambient glycemia in mice on HFD (Figure 1B, right). This could be explained by a concurrent decrease in glucose clearance, which we then calculated. While acute 9-PAHSA infusion did not affect glucose clearance in chow-fed mice, it reduced glucose clearance in HFD-fed mice (Figure 1C) at the same time that it reduced EGP. This result explains the lack of change in glycemia with 9-PAHSA infusion in HFD-fed mice.
Insulin levels were elevated in both vehicle- and PAHSA-treated HFD-fed mice compared with chow-fed mice, reflecting their insulin resistance, and acute PAHSA infusion did not change ambient serum insulin levels on either diet (Figure 1D). Fasting induces hormones such as glucagon that increase hepatic glucose production by increasing cAMP and activating PKA, which phosphorylates target proteins such as cAMP response element binding protein (CREB) (28). Therefore, we aimed to determine whether the acute effect of 9-PAHSA on EGP is due to attenuation of the PKA signaling cascade. Figure 1E shows that 9-PAHSA infusion reduced CREB phosphorylation by $48 \%$ in liver. In addition, 9-PAHSA infusion decreased the expression of 2 CRE-containing genes, glucose-6 phosphatase catalytic subunit (G6pc1) and phosphoenolpyruvate carboxykinase 1 (Pck1), by 66\% and 32\%, respectively, in chow-fed mice (Figure $1 \mathrm{~F}$ ), consistent with effects on PKA signaling. Moreover, 9-PAHSA infusion reduced G6pc1 mRNA levels by $76 \%$ in mice on an HFD, similar to the results in chow-fed mice (Figure 1F). 9-PAHSA infusion also decreased hepatic glucose-6-phosphatase (G6pase) maximal activity in liver of both chow- and HFD-fed mice, without changing $K_{m}$ (Figure $1 G)$. G6pase is rapidly regulated by cAMP (29) and catalyzes the rate-limiting step of glucose output, which exerts greater control than Pck1 on the short-term regulation of EGP $(30,31)$. Taken together, these data indicate that acute PAHSA administration decreases EGP through inhibition of the cAMP/PKA signaling pathway, which leads to decreased G6pase activity.

Chronic PAHSA treatment decreases glycemia during pyruvate and lactate tolerance test in chow- and HFD-fed mice. To determine whether chronic PAHSA treatment can prevent the insulin resistance that is characteristic of HFD feeding, we delivered 5-PAHSA $(0.1 \mathrm{mg} / \mathrm{d})$ and 9-PAHSA $(0.4 \mathrm{mg} / \mathrm{d})$ via subcutaneous osmotic minipumps starting with the initiation of HFD feeding. We selected 9- and 5-PAHSA because 9-PAHSA is the most abundant isomer in WAT and brown adipose tissue (BAT) in WT mice and is also downregulated in WAT of insulin-resistant humans (13); and 5-PAHSA is the most consistently downregulated isomer in serum and all adipose depots of insulin-resistant mice and in WAT and serum of insulin-resistant humans (13). Since serum levels of 9-PAHSA are 3-fold higher than those of 5-PAHSA (13), we administrated 9- and 5-PAHSA at a 3:1 ratio. PAHSA treatment had no effect on body weight (Figure 2A), fat mass, or food intake (Supplemental Figure 2 , A and B), consistent with our previous study, in which we treated mice that had already been on an HFD with PAHSAs (18). In HFDfed mice, PAHSA treatment did not change glucagon levels after 5-hour food removal (Supplemental Figure 2C). In chow-fed mice, serum 5-PAHSA levels increased 3.5-fold and 9-PAHSA levels 2.5fold relative to those in vehicle-treated mice, and in HFD-fed mice 5-PAHSA levels increased 7-fold and 9-PAHSA levels 3.7-fold relative to chow/vehicle levels (Figure 2B). The greater fold increase in HFD-fed mice most likely reflects the higher treatment dose compared with our previous study in mice already on an HFD (18). Consistent with the effects observed with a single dose of PAHSAs (Supplemental Figure 1), chronic PAHSA treatment also decreased glycemia during pyruvate and lactate challenge in 18-hour-fasted chow- and HFD-fed mice (Supplemental Figure 3).

Chronic PAHSA treatment increases hepatic and systemic insulin sensitivity in chow-fed mice. To examine whether the inhibitory 

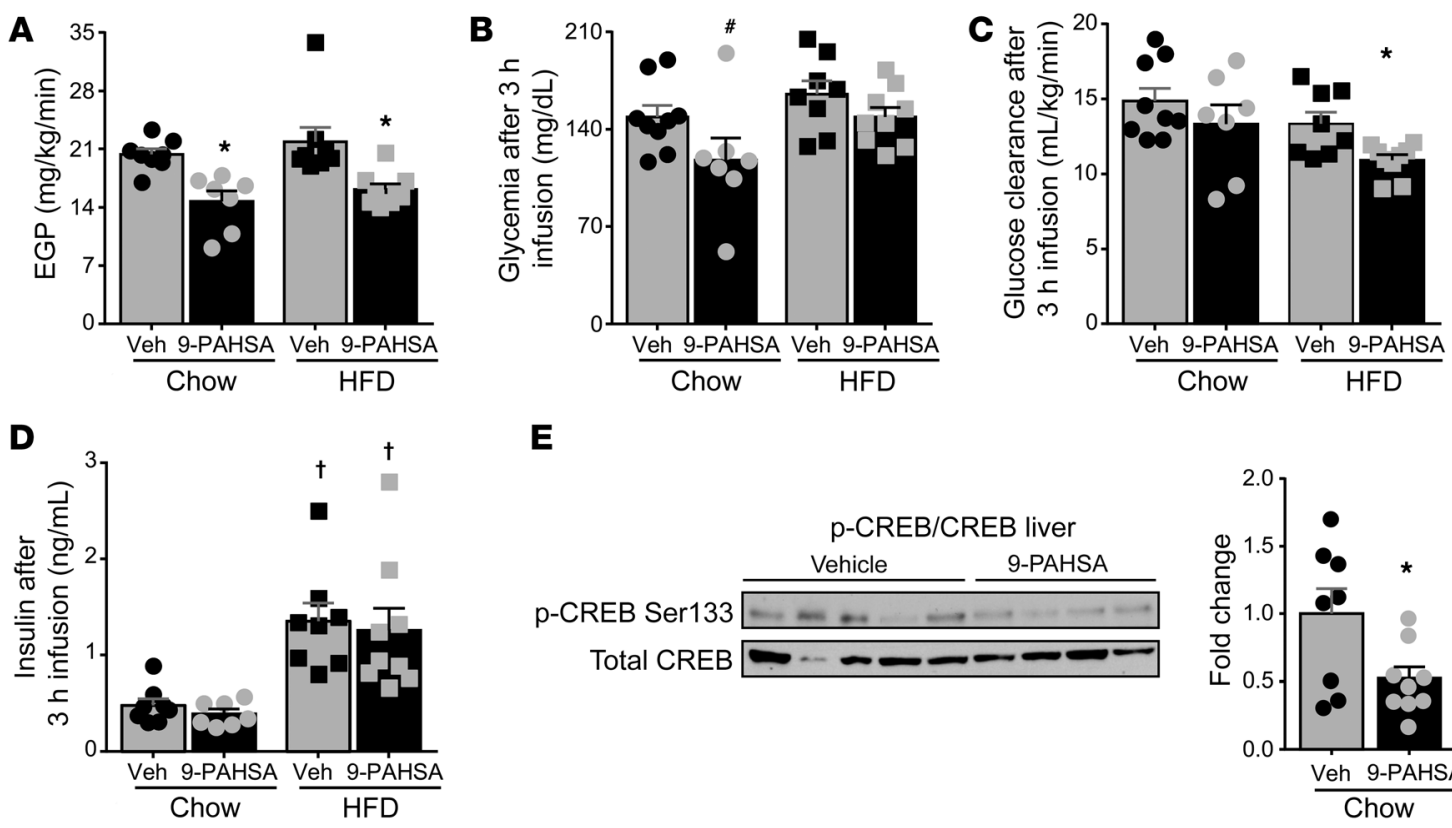

E
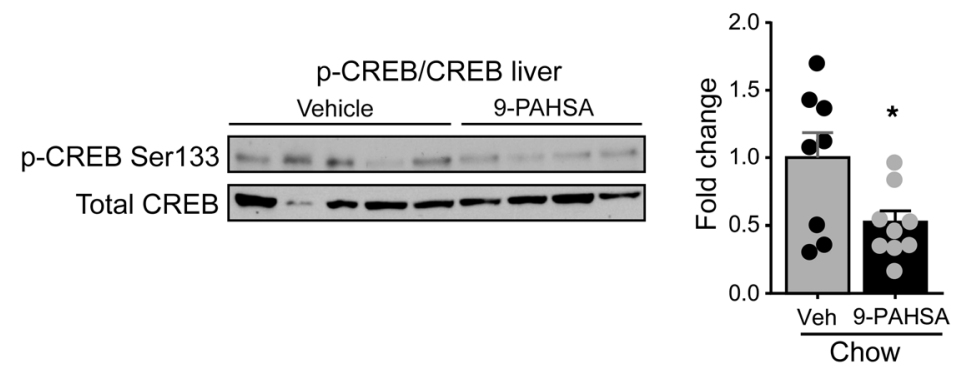

$\mathbf{F}$

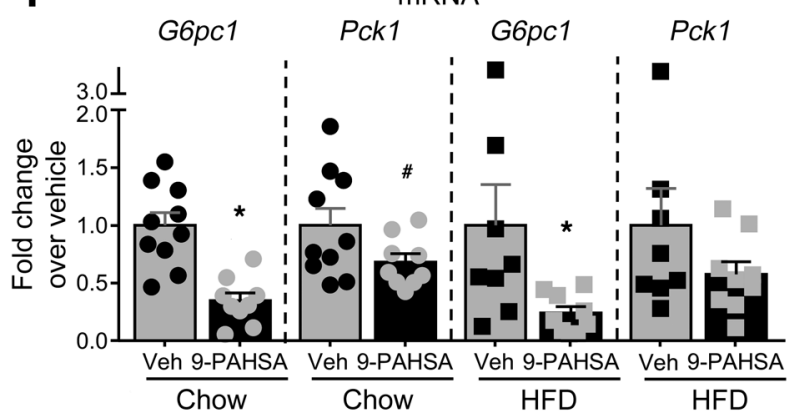

G

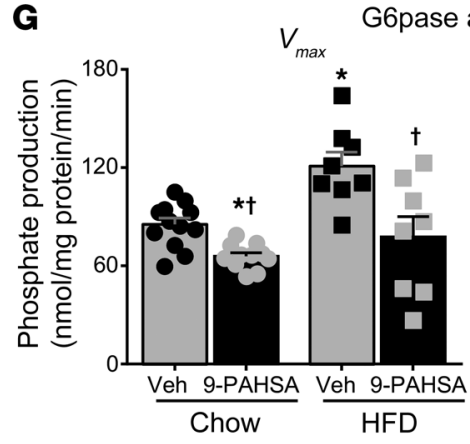

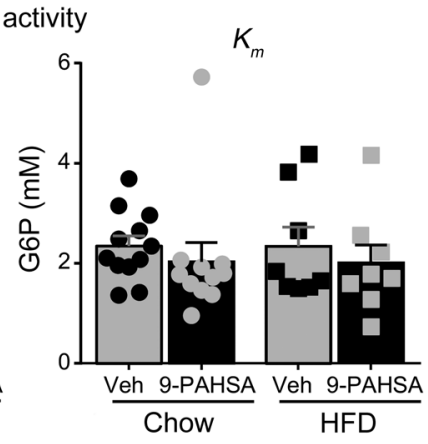

Figure 1. Acute PAHSA treatment decreases glucose production in vivo through inhibition of cAMP signaling. Five hours after food removal, [3- ${ }^{3} \mathrm{H}$ ] glucose $(0.05 \mu \mathrm{Ci} / \mathrm{min})$ was infused through the jugular vein together with vehicle (Veh) $(0.5 \% \mathrm{BSA}, 3 \mu \mathrm{L} / \mathrm{h})$ or 9-PAHSA (chow: $9 \mu \mathrm{g} / \mathrm{h}$; HFD: $18 \mu \mathrm{g} / \mathrm{h}$, preconjugated with $0.5 \%$ BSA) for 3 hours in 16-week-old mice on a chow or HFD for 11 weeks. Endogenous glucose production (EGP) (A), glycemia (B), glucose clearance (C), and insulin levels (D) at the end of infusion. $n=7-9 /$ group. (E) Representative Western blots for total and phosphorylated CREB proteins in liver lysates from chow-fed mice at the end of infusion. $n=8-9 / g r o u p$. Bar graph represents fold change relative to vehicle. This Western blot is representative of 4 Western blots using the same samples. (F) Hepatic glucose-6-phosphatase catalytic unit (G6pc1) and phosphoenolpyruvate carboxykinase 1 (Pck1) mRNA expression in chow- and HFD-fed mice at the end of the infusion. G6pc1 and Pck1 mRNA are expressed as a ratio to GAPDH, which was used as the housekeeping gene. Graphs represent fold change relative to vehicle. $n=8-10 /$ group. $(\mathbf{A}-\mathbf{F}){ }^{*} P<0.05$ versus vehicle, ${ }^{*} P=0.08$ versus chow vehicle, ${ }^{\dagger} P<0.05$ versus chow diet. (C) Hepatic glucose- 6 -phosphatase (G6pase) activity $\left(V_{\max }\right.$ and $\left.K_{m}\right)$ at the end of the infusion. $n=8-11 /$ group. ${ }^{*} P<0.05$ versus vehicle-treated mice on a chow diet, ${ }^{\dagger} P<0.05$ versus vehicle-treated HFD-fed mice. Statistical significance for all panels was evaluated by 2 -way ANOVA, followed by Tukey's post hoc test or unpaired 2-tailed Student's $t$ test. Data are mean \pm SEM.

effect of PAHSAs on hepatic gluconeogenesis is associated with an improvement in systemic insulin sensitivity, we performed hyperinsulinemic-euglycemic clamps (insulin infusion rate, 2.5 $\mathrm{mU} / \mathrm{kg} / \mathrm{min}$ ) in chow-fed mice chronically treated with PAHSAs or vehicle. During the clamp period, blood glucose levels were maintained at approximately $120 \mathrm{mg} / \mathrm{dL}$ in both groups (Figure 2C). Insulinemia was increased to comparable levels (Supplemental Figure 4A). Compared with vehicle-treated mice on a chow diet, PAHSA-treated mice needed a significantly higher glucose infusion rate (GIR) to maintain euglycemia, indicating that chronic PAHSA treatment improves insulin sensitivity (Figure 2D). These results reinforce our previous data showing improvements in glucose tolerance and insulin sensitivity, measured during glucose and insulin tolerance tests, in mice chronically treated with PAHSAs (18). To determine which tissues contribute to the improved insulin sensitivity, we analyzed the tracer data to assess the discrete components of basal and insulin-stimulated glucose metabolism. In the basal state, in chow-fed mice treated with PAHSAs, basal glucose clearance tended to be lower (Figure 2E) and basal EGP was lower compared with that in vehicle-treated mice (Figure 2F). In addition, insulin suppression of EGP was markedly greater in PAHSA-treated mice, indicating that PAHSA treatment enhances hepatic insulin sensitivity (Figure 2F).

Furthermore, the glucose disappearance rate (Rd) was 30\% higher in PAHSA-treated compared with vehicle-treated chow-fed mice (Figure 2G). However, 2-[1-14 C]-deoxy-D-glucose (2-DOG) uptake into individual muscles and adipose depots was not different between vehicle- and PAHSA-treated mice with a $2.5 \mathrm{mU}$ / 
A

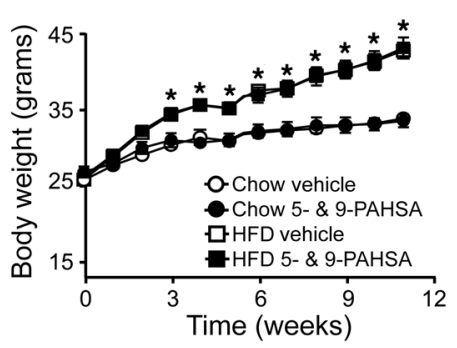

C

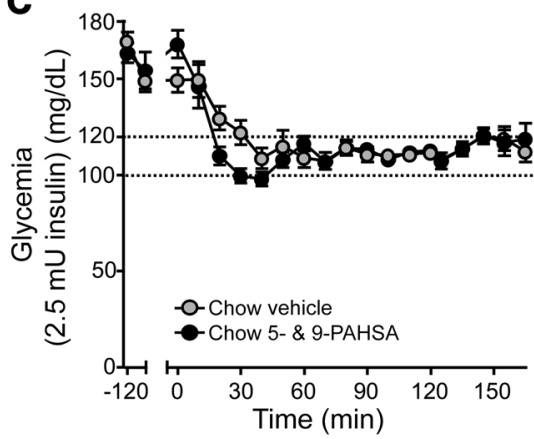

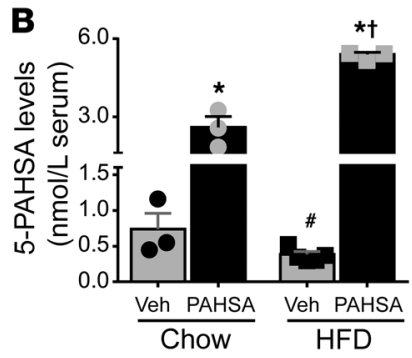

D

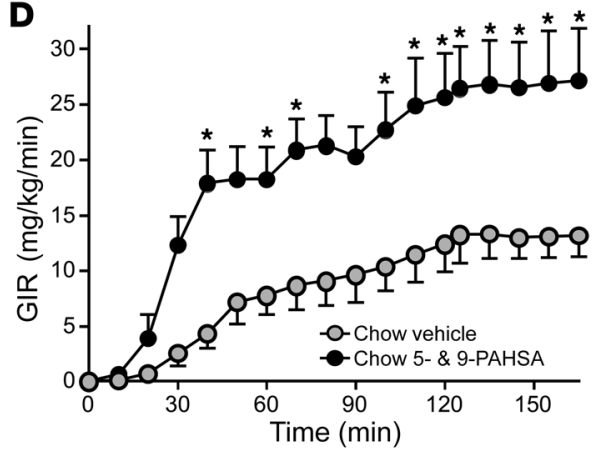

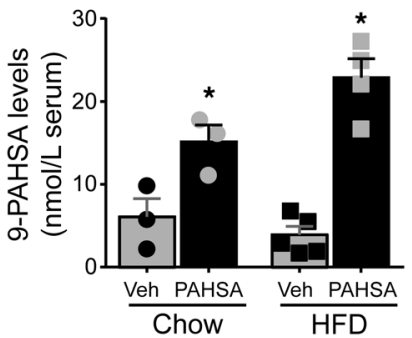

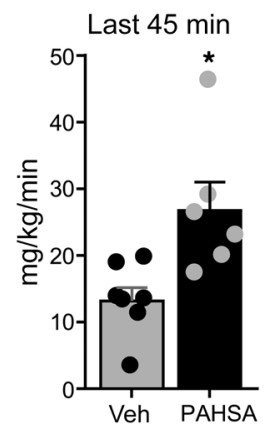

E

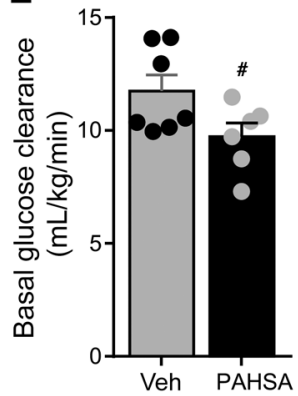

$\mathbf{F}$
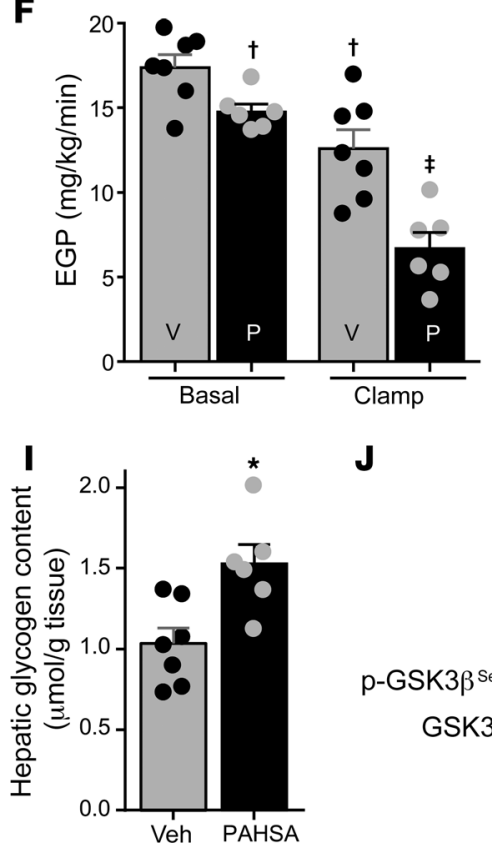

J

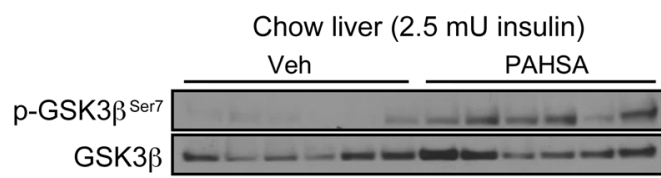

G

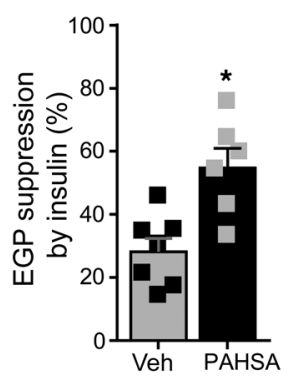

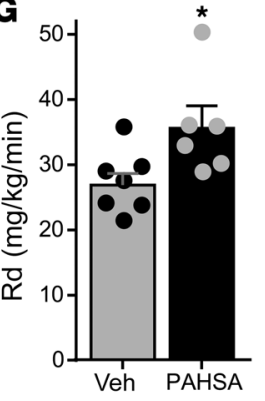

H

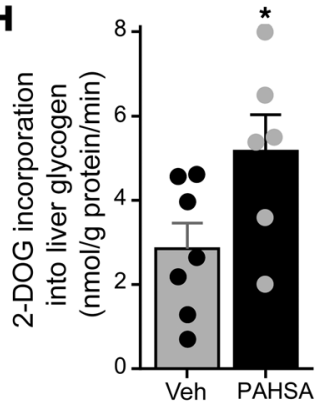

Figure 2. Chronic PAHSA treatment increases systemic insulin sensitivity in chow-fed mice. (A) Body weight of C57BL/6 male mice treated with 5-PAHSA $(0.1 \mathrm{mg} / \mathrm{d})$ and 9-PAHSA $(0.4 \mathrm{mg} / \mathrm{d})$ delivered by subcutaneous osmotic minipumps. PAHSA treatment was started with the initiation of HFD feeding at 12 weeks of age. Chow: $n=10$ /group; HFD: $n=20$ /group. ${ }^{*} P<0.05$ for both HFD groups versus both chow-fed mice. (B) Serum 5-PAHSA (left) and 9-PAHSA (right) levels at 2 months of PAHSA treatment. $n=3-5$ /group (each sample represents a pool of serum from 3-4 mice). ${ }^{*} P<0.05$ versus vehicle-treated mice, ${ }^{\#} P=0.08$ versus vehicle-treated mice on a chow diet, ${ }^{\dagger} P<0.05$ versus PAHSA-treated mice on a chow diet. (C-C) Hyperinsulinemiceuglycemic clamp studies were performed with a $2.5-\mathrm{mU} / \mathrm{min} / \mathrm{kg}$ insulin infusion rate after 5 -hour food removal in chow mice treated with PAHSAs or vehicle for 13 weeks: glycemia (C), Glucose infusion rate (GIR) and GIR at steady state (bar graph) (D), basal glucose clearance (E), EGP and percent suppression of EGP by insulin (F), and disappearance rate (Rd) $(\mathbf{C}) . n=6-7 /$ group. (H-K) 2-[1-14C]-deoxy-D-glucose (2-DOG) incorporation into liver glycogen (H), levels of liver glycogen (I), hepatic GSK3 $\beta$ signaling (representative of 3 Westerns blots using same samples) (J), and liver triglyceride (TC) levels (K) at the end of the clamp. $n=6-7 /$ group. For $\mathbf{C}-\mathbf{K},{ }^{*} P<0.05$ versus vehicle-treated chow mice, ${ }^{*} P=0.06$ versus vehicle, ${ }^{\dagger} P<0.05$ versus vehicle-treated chow mice at baseline, ${ }^{\ddagger} P<0.05$ versus all other groups. Statistical significance for $\mathbf{A}, \mathbf{C}$, and $\mathbf{D}$ was evaluated by 2 -way repeated-measures ANOVA; for all others, 1 - or 2-way ANOVA followed by Tukey's post hoc test or unpaired 2-tailed Student's $t$ test. Data are mean \pm SEM. 
$\mathrm{kg} / \mathrm{min}$ insulin infusion rate (Supplemental Figure 4B). Therefore, we investigated whether hepatic glucose uptake could contribute to the difference in $\mathrm{Rd}$ (32). Hepatic glucose uptake cannot be precisely measured with the 2-DOG uptake technique owing to the presence of G6pase in the liver, which regulates both glucose uptake and output so that 2-DOG is not "trapped" in liver as it is in muscle and adipocytes. However, 2-DOG incorporation into glycogen in liver provides some indication of hepatic glucose uptake $(33,34)$. The rate of 2-DOG incorporation into glycogen in PAHSA-treated mice was increased $80 \%$ compared with that in vehicle-treated mice, suggesting an increase in hepatic glucose uptake (Figure $2 \mathrm{H}$ ). Consistent with this, chronic PAHSA treatment increased liver glycogen content by $50 \%$ (Figure 2I), and there was a similar tendency with acute 9-PAHSA infusion in chow-fed mice (data not shown). To investigate the mechanisms underlying PAHSA-driven improvement of hepatic insulin sensitivity, we measured the effects of PAHSAs on hepatic insulin signaling at the end of the clamp. PAHSA treatment increased the phosphorylation and the total amount of Akt in liver of chow-fed mice. Although the p-Akt/Akt ratio was unchanged (Supplemental Figure 5), there was effectively more p-Akt with PAHSA treatment. This is reflected in increased phosphorylation of the AKT target protein glycogen synthase kinase $3 \beta$ (GSK3 $\beta$ ) in PAHSA-treated chow mice (Figure 2J). These data suggest that enhanced hepatic insulin signaling in PAHSA-treated chow mice could contribute to increased hepatic glycogen content and hepatic insulin sensitivity. Inhibition of hepatic glucose production can divert carbons toward lipid synthesis, leading to triglyceride (TG) accumulation in the liver and ultimately hepatic steatosis. However, chronic PAHSA treatment did not change liver TG levels in chow-fed mice (Figure 2K).

Chronic PAHSA treatment improves hepatic and systemic insulin sensitivity in insulin-resistant HFD-fed mice. To determine whether PAHSA treatment improves hepatic insulin resistance in HFD-fed mice, we initially used an insulin infusion rate of $2.5 \mathrm{mU} / \mathrm{kg} / \mathrm{min}$. Euglycemia was maintained during the clamp in both the vehicleand PAHSA-treated groups (Figure 3A), along with comparable increases in insulinemia in the groups (Supplemental Figure 6A). Chronic PAHSA treatment resulted in a marked improvement of whole-body insulin sensitivity, indicated by a 4.5 -fold increase in GIR (Figure 3B). Chronic PAHSA treatment did not affect basal EGP in HFD-fed mice (Figure $3 \mathrm{C}$ ). However, in vehicle-treated HFD-fed mice, insulin at this infusion rate did not suppress EGP, whereas PAHSA treatment resulted in a $37 \%$ suppression of EGP, indicating that PAHSAs improve hepatic insulin sensitivity (Figure 3C). Rd, 2-DOG uptake in muscle and fat depots, and liver 2-DOG incorporation into glycogen were not different between the HFD groups at this insulin infusion rate (Supplemental Figure 6, B-D). Liver TG levels were not changed after chronic PAHSA treatment in HFD-fed mice (Figure 3D).

Since the $2.5 \mathrm{mU} / \mathrm{kg} / \mathrm{min}$ insulin infusion rate is optimal for assessing hepatic insulin sensitivity but may be insufficient for analyzing peripheral insulin sensitivity in insulin-resistant mice (35), we performed a second hyperinsulinemic-euglycemic clamp in the HFD-fed mice with an insulin infusion rate of $4 \mathrm{mU} / \mathrm{kg} / \mathrm{min}$. Euglycemia was also reached in both HFD groups (Figure 3E), and insulinemia during the clamp period was not different between PAHSA- and vehicle-treated mice (Supplemental Figure 6E).
PAHSA-treated HFD mice required a significantly higher GIR to maintain euglycemia than vehicle-treated HFD mice also with this higher insulin infusion rate (Figure 3F). In addition, the PAHSAtreated mice showed greater suppression of EGP by insulin (Figure $3 G$ ), even though this higher insulin infusion rate also suppressed EGP more in vehicle-treated mice than the lower infusion rate. Rd was not different between vehicle- and PAHSA-treated HFD-fed mice at the $4 \mathrm{mU} / \mathrm{kg} / \mathrm{min}$ insulin infusion rate (Figure $3 \mathrm{H})$. However, PAHSA treatment increased glucose uptake in the extensor digitorum longus (EDL) muscle and heart, although not into other muscles (anterior tibialis, soleus, or gastrocnemius) (Figure 3I and Supplemental Figure 6F). Chronic PAHSA treatment also increased Akt phosphorylation and the p-Akt/Akt ratio in heart, EDL, and liver at the end of the clamp in HFD-fed mice (Figure 3J). Phosphorylation of GSK3 $\beta$ was highly variable, and the p-GSK3 $\beta /$ GSK3 $\beta$ ratio was not different between vehicle and PAHSA treatments in any of the tissues analyzed (Supplemental Figure 7). Together, these data indicate that enhanced Akt signaling in heart, EDL, and liver may contribute to improved systemic insulin sensitivity in PAHSA-treated HFD-fed mice, although the impact of PAHSAs on specific signaling molecules differed from that in chow-fed mice (Figure 2J). Liver glycogen content was 4-fold higher in HFD-fed mice (Figure 3K) compared with chowfed mice (Figure 2I), as previously reported (36). Interestingly, chronic PAHSA treatment markedly decreased liver glycogen content in HFD-fed mice to levels similar to those in PAHSA-treated chow-fed mice. This was not observed with acute PAHSA infusion (data not shown).

PAHSAs suppress lipolysis, and this partially accounts for the effects on suppression of EGP. Dysregulation of WAT lipolysis can lead to increased hepatic gluconeogenesis by two mechanisms: (i) release of glycerol provides the liver with an increased substrate for conversion to glucose through a substrate push mechanism; and (ii) increased acetyl-CoA delivery to the liver activates liver pyruvate carboxylase, which catalyzes an important early step in gluconeogenesis (5). Both processes result in hepatic insulin resistance (5). To determine whether PAHSAs could enhance hepatic insulin sensitivity through effects on lipolysis, we measured serum FFA levels. In chow-fed mice, FFA levels at the end of the clamp were lower in PAHSA-treated compared with vehicle-treated mice (Figure 4A). In HFD-fed mice, FFA concentrations were similar at the beginning of the clamp but lower at the end of the clamp in PAHSA-treated mice compared with vehicle-treated mice, indicating that PAHSAs increase sensitivity to insulin suppression of plasma FFA concentrations (Figure 4A). This suggests that PAHSAs increase adipose tissue sensitivity to the antilipolytic effects of insulin and that this may contribute to the hepatic and systemic insulin-sensitizing effects of PAHSAs. To test whether PAHSAs have a direct effect on inhibition of lipolysis, we carried out experiments in murine adipose tissue explants. Treatment with the $\beta$-adrenergic agonist isoproterenol increased lipolysis in adipose tissue explants, and 9-PAHSA inhibited isoproterenol-induced lipolysis with effects similar to those of insulin (Figure 4B). The direct effects of PAHSAs and insulin in WAT explants did not appear to be additive (Figure 4B and Supplemental Figure 8B). In addition, 9-PAHSA suppressed lipolysis in perigonadal WAT, but not in SQWAT (Supplemental Figure 8B). 

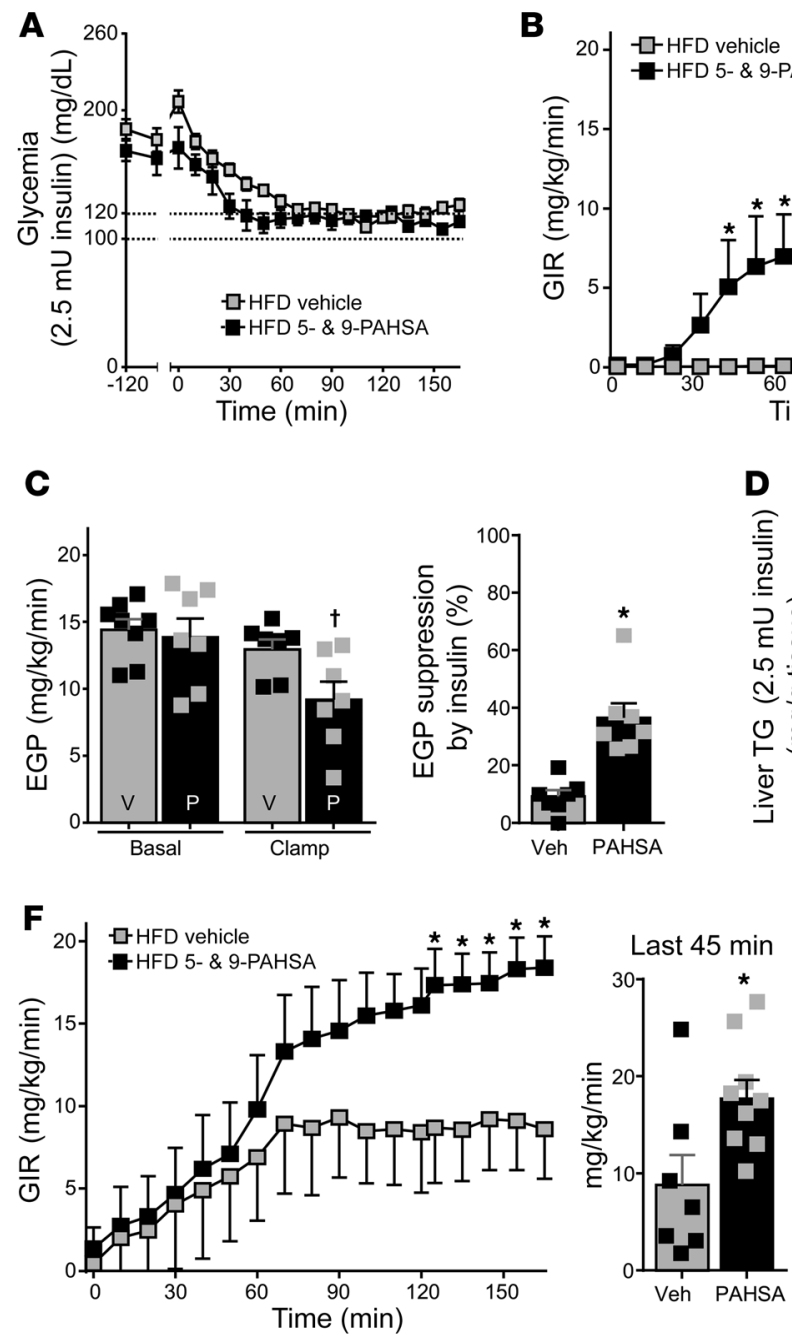
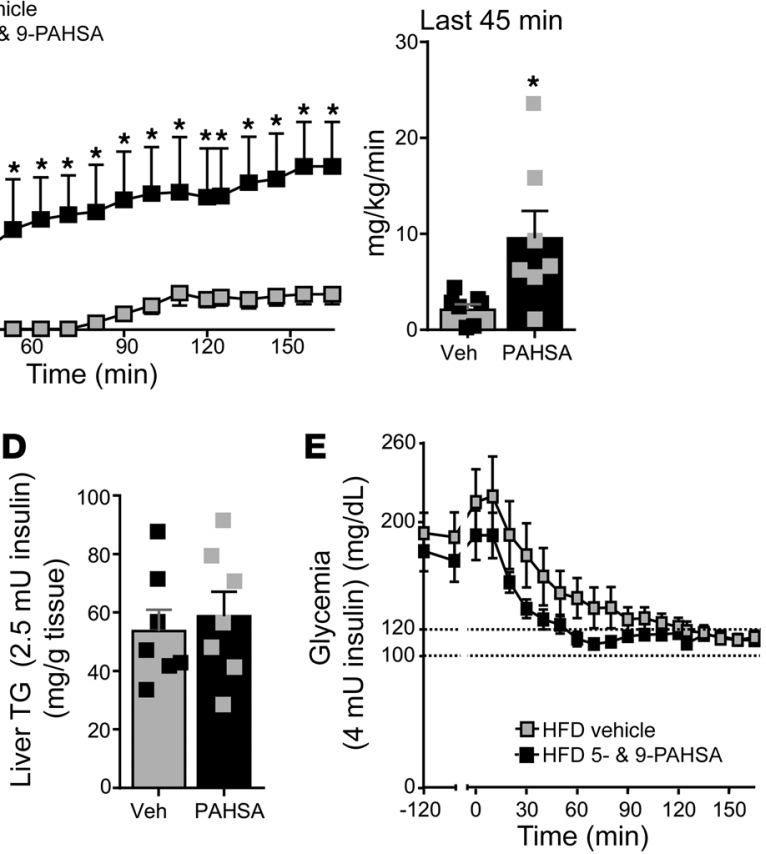

G

H
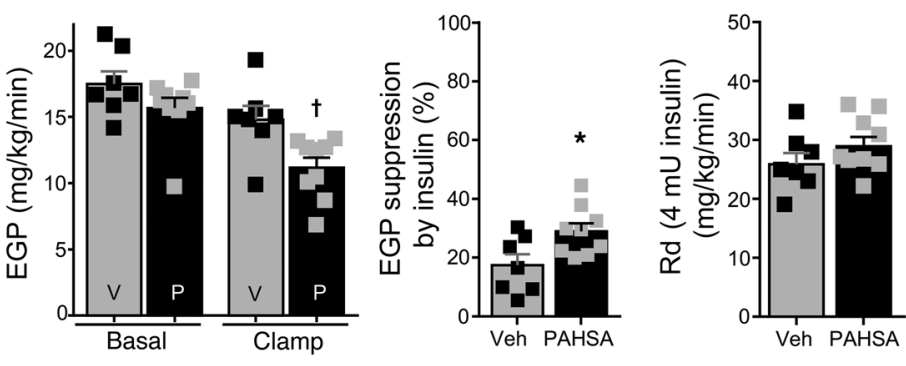

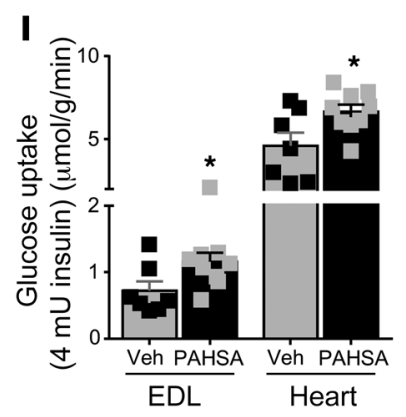

K

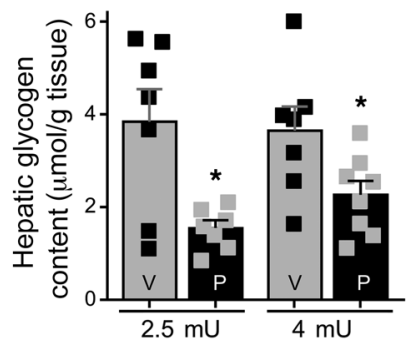

$\mathbf{J}$

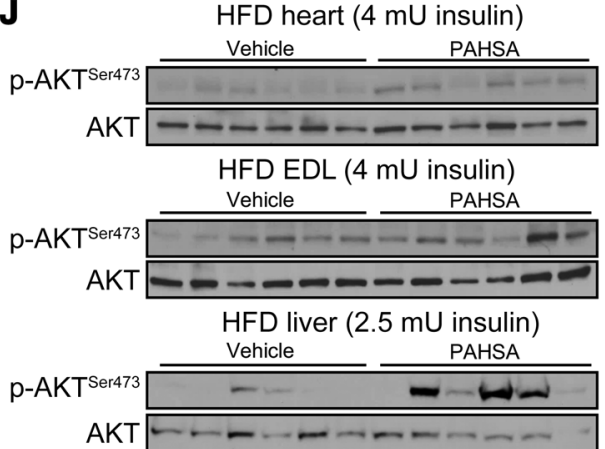

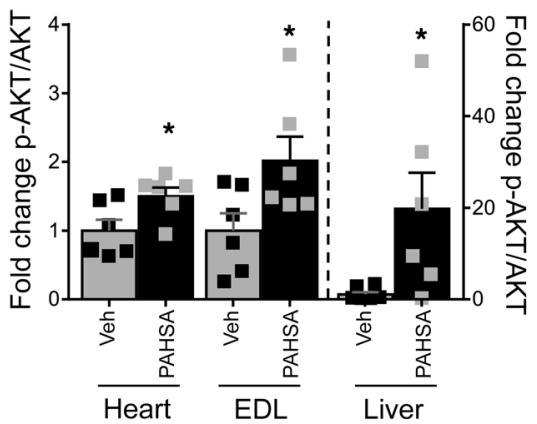

Figure 3. Chronic PAHSA treatment improves hepatic and systemic insulin sensitivity in insulin-resistant HFD-fed mice. (A-C) Hyperinsulinemic-euglycemic clamp studies were performed with $2.5 \mathrm{mU} / \mathrm{min} / \mathrm{kg}$ insulin infusion rate after 5-hour food removal in HFD-fed mice treated with 5- and 9-PAHSAs or vehicle for 13 weeks. Glycemia (A), GIR and GIR at steady state (bar graph) (B), and EGP and percent suppression by insulin of EGP (C). $n=7$ /group. (D) Liver TC levels at the end of the $2.5 \mathrm{mU}$ clamp. $n=7 /$ group. (E-H) Hyperinsulinemic-euglycemic clamp studies were performed with a $4-\mathrm{mU} / \mathrm{min} / \mathrm{kg}$ insulin infusion rate after 5 -hour food removal in HFD-fed mice treated with 5- and 9-PAHSAs or vehicle for 13 weeks. Glycemia (E), GIR and GIR at steady state (F), EGP and percent suppression by insulin of EGP (G), and Rd (H). $n=7-9$ /group. (I) In vivo tissue 2-DOC uptake at the end of the 4-mU clamp. $n=7-9$ /group. (J) Akt signaling in heart, extensor digitorum longus muscle (EDL), and liver. $n=6$ /group. (K) Hepatic glycogen content at the end of the $2.5-\mathrm{mU}$ and $4-\mathrm{mU}$ clamps. $n=7-8 /$ group. ${ }^{*} P<$ 0.05 versus vehicle-treated HFD-fed mice, ${ }^{\dagger} P<0.05$ versus all other groups. Statistical significance for A, B, E, and $\mathbf{F}$ was evaluated by 2-way repeated-measures ANOVA; for all others, 2-way ANOVA followed by Tukey's post hoc test or unpaired 2-tailed Student's $t$ test. Data are mean \pm SEM. 
To determine whether PAHSAs' effects on lipolysis are required for their suppression of EGP, we performed hyperinsulinemic-euglycemic clamps with concurrent Intralipid infusion to prevent the suppression of FFAs and glycerol in chronic vehicle- or PAHSA-treated mice. Plasma FFAs were elevated approximately 2- to 3-fold during Intralipid infusion (Supplemental Figure 9, A and B) in both chowand HFD-fed mice. As expected, Intralipid infusion for 2 hours did not alter GIR in vehicle-treated mice on a chow diet (6), but tended to prevent the increase in GIR in PAHSA-treated mice (Figure 4C), although it did not affect the suppression of EGP driven by PAHSAs in mice on a chow diet (Figure 4D). PAHSA-treated mice receiving Intralipid showed higher insulinemia at the end of clamp (Supplemental Figure 9C), although no differences in the fold increase over basal insulinemia were found among the experimental groups (Supplemental Figure 9D). Conceivably, the insulin levels were higher at the end of clamp in PAHSA-treated mice receiving Intralipid because these animals had higher starting insulinemia (Supplemental Figure 9C). However, this was not due to differences in endogenous insulin secretion, since C-peptide levels are comparable among the experimental groups both before and at the end of the clamp (Figure 4E). On the other hand, in mice on an HFD, in which serum insulin levels were unchanged between treatments (Figure 4G), Intralipid infusion prevented the insulin-sensitizing effect of PAHSAs on GIR and EGP (Figure 3, F and G, and Figure 4, F and H). This suggests that the antilipolytic effect of PAHSAs is responsible for the improvement of hepatic insulin sensitivity in HFD-fed mice.

PAHSAs directly inhibit basal and glucagon-stimulated EGP through a cAMP-dependent pathway involving $G \alpha_{i}$ protein-coupled receptors. Since Intralipid infusion fails to block the effects of PAHSAs on EGP in chow-fed mice, we investigated whether PAHSAs have a direct effect on EGP in isolated hepatocytes. A 3-hour treatment with 5- and 9-PAHSA inhibited basal EGP by $34 \%$ and $49 \%$, respectively, in primary hepatocytes (Supplemental Figure 10 and Figure 5A), and this effect was not additive with insulin at maximal or submaximal concentrations (Figure $5 \mathrm{~A}$ ). Furthermore, 9-PAHSA decreased glucagon-stimulated EGP dose dependently in primary hepatocytes (Figure 5B). Consistent with the data demonstrating that an acute PAHSA infusion attenuated PKA signaling in vivo (Figure $1, \mathrm{E}-\mathrm{G}$ ), we found that 30 minutes of 9-PAHSA incubation reduced glucagon-stimulated cAMP levels by approximately $39 \%$ in isolated hepatocytes (Figure 5C). Furthermore, 9-PAHSA did not alter 8-bromo-cAMP-stimulated EGP (Figure 5D), indicating that 9-PAHSA acts upstream of PKA.

Our previous studies have demonstrated that some of the biological functions of PAHSAs are mediated through $\mathrm{G}$ protein-coupled receptors (GPCRs) $(13,18)$. Here we show that the effects of 9-PAHSAs on basal and glucagon-stimulated EGP were abolished by pertussis toxin (PTX) (Figure 5E), which inactivates $\mathrm{G}_{\mathrm{i}}$-type GPCRs. Together, these data indicate that the effects of PAHSAs on EGP appear to be mediated by a cAMP-dependent pathway involving $G \alpha_{i}$ protein-coupled receptors (Figure 5F). Consistent with this, PAHSA-mediated inhibition of lipolysis in adipose explants was also blocked by PTX (data not shown).

\section{Discussion}

EGP regulation is a fundamental component of glucose homeostasis, and impaired insulin-mediated suppression of EGP plays a major role in the pathogenesis of T2D and its complications (37). In this article, we show that PAHSA treatment markedly increases insulin action to suppress EGP in aged chow- and HFD-fed mice. In addition, in HFD-fed mice, chronic PAHSA treatment improves insulin-stimulated glucose uptake in glycolytic muscle and heart. We found that the mechanisms by which PAHSAs enhance hepatic insulin sensitivity involve direct and indirect effects, including intertissue communication between adipose tissue and liver. Suppression of lipolysis appears to be a key mechanism for the insulin-sensitizing effects of PAHSAs in HFD-fed mice. Our data suggest that PAHSAs might reduce the delivery of gluconeogenic substrates to the liver by inhibiting WAT lipolysis in HFD-fed mice, resulting in reduced EGP. In contrast to our previous chronic PAHSA treatment studies (18), in the current study we used a disease prevention design in which we started PAHSA treatment and HFD simultaneously. Thus, this study shows that PAHSAs can prevent the development of insulin resistance with aging in chowand HFD-fed mice. Hence, these bioactive lipids might be developed into novel therapeutic agents for T2D prevention.

The relative importance of the direct and indirect mechanisms for EGP regulation by insulin is still under investigation (4-7). The direct effect of insulin has been shown to be sufficient to suppress EGP in experimental conditions mimicking the postprandial rise in hepatic insulinemia (4). This effect results from increased insulin signaling, which increases glycogen synthesis and reduces hepatic glucose output. In our study, we found increased insulin signaling in the liver of PAHSA-treated mice. This could be secondary to the increased insulin sensitivity resulting from chronic PAHSA treatment, because we have not observed any change in insulin signaling in PAHSA-treated mice in response to acute insulin injection in vivo or in PAHSA-treated isolated hepatocytes in response to shortterm incubation with insulin. With prolonged incubation of HepG2 cells with 5-PAHSA, insulin signaling was increased (27), consistent with our data with chronic PAHSA treatment. But under different conditions, other mechanisms including the indirect pathway (i.e.,suppression of lipolysis) play a major role (5). Several groups have demonstrated the importance of the indirect effects of insulin in suppressing EGP using genetic models of ablation of hepatic insulin signaling (i.e., liver Akt1-, Akt2-, and Foxo1-KO mice) and also HFD-induced insulin resistance $(5,6,38)$.

PAHSAs enhance sensitivity to insulin for some actions in adipose tissue, such as glucose transport and translocation of Glut4 glucose transporters from intracellular vesicles to the plasma membrane $(13,19)$. Here we show that PAHSAs inhibit lipolysis directly in WAT explants ex vivo. PAHSAs also enhance the antilipolytic effect of insulin in vivo, as indicated by a greater reduction in FFAs at the end of the clamp in PAHSA-treated mice. Given their antilipolytic effect, we investigated whether inhibition of WAT lipolysis is critical in order for PAHSAs to improve hepatic and systemic insulin sensitivity. To test this, we clamped FFA and glycerol levels using Intralipid infusion, and this blocked the effects of PAHSAs on hepatic insulin sensitivity in HFD-fed mice. However, Intralipid infusion did not block the effects of PAHSAs on EGP in chow-fed mice, potentially because the degree of insulin resistance and the amount of lipolysis are lower in chow-fed mice. In addition, in WT mice on a chow diet, the inhibition of WAT lipolysis is not the main mechanism mediating the suppression of EGP by insulin, as demonstrat- 
A

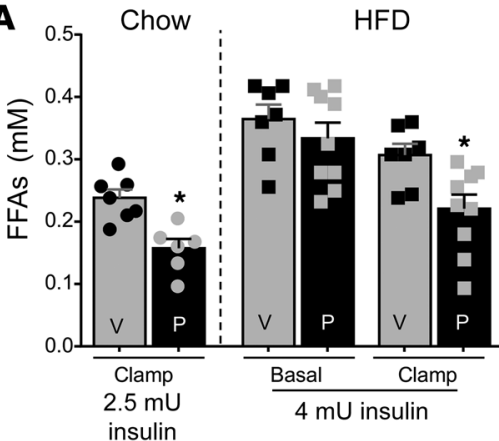

B

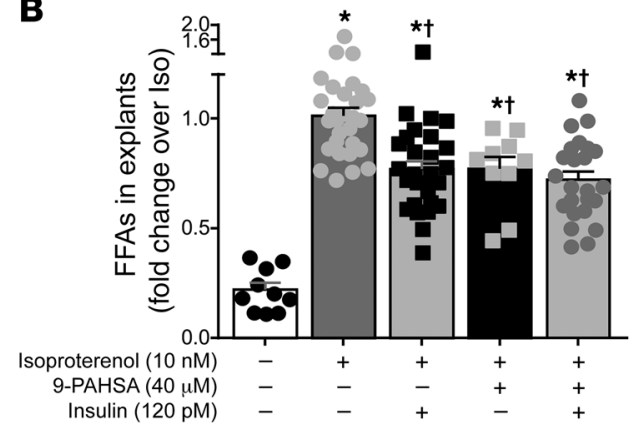

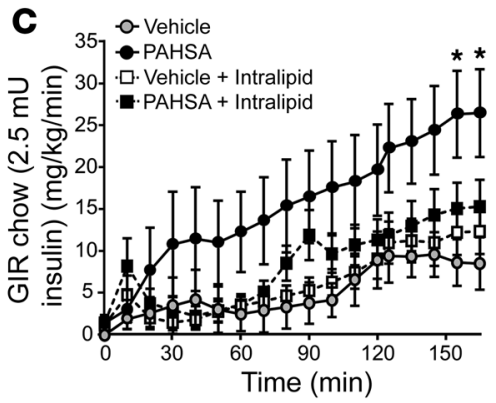

D

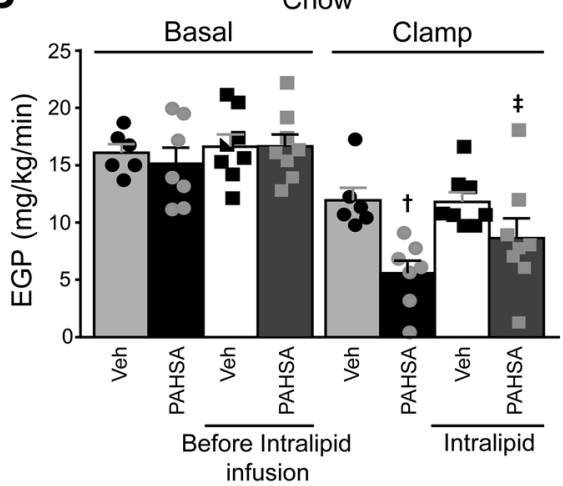

Last $45 \mathrm{~min}$

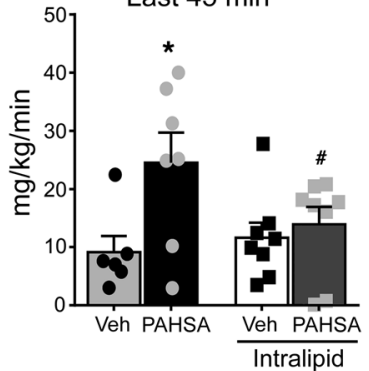

E

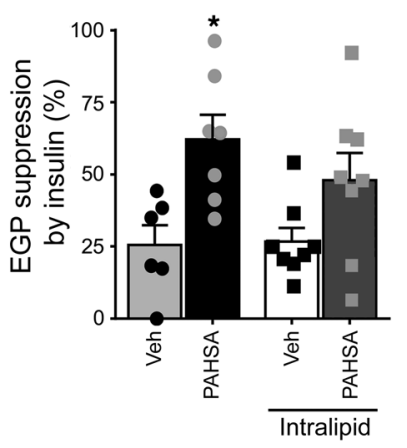

G

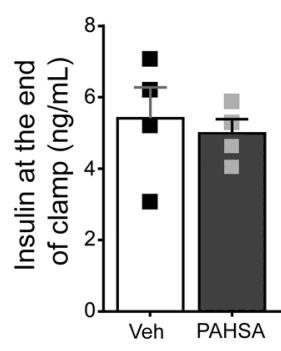

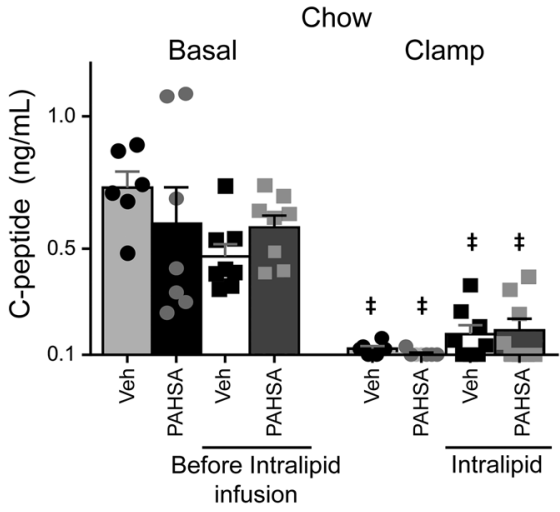
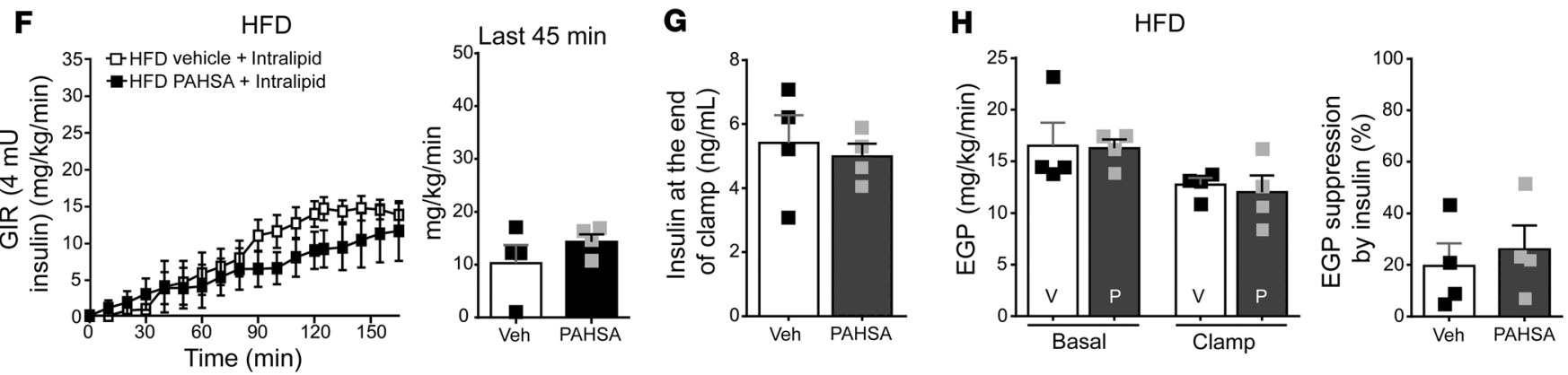

Figure 4. PAHSAs suppress lipolysis, and this partially accounts for the effects of PAHSAs to suppress EGP. (A) Free fatty acid (FFA) levels at baseline and at the end of the clamp. Insulin dose: chow, $2.5 \mathrm{mU} / \mathrm{kg} / \mathrm{min}$ (Figure 2, C-G); HFD, $4 \mathrm{mU} / \mathrm{kg} / \mathrm{min}$ (Figure $3, \mathrm{E}-\mathrm{H}$ ). $n=6-9 /$ group. ${ }^{*} P<0.05$ versus all other groups within the same diet. (B) FFA release from perigonadal WAT explants. $n=10-29$ /group. ${ }^{*} P<0.05$ versus untreated control cells, ${ }^{\dagger} P<0.05$ versus isoproterenol-treated cells. (C-H) Hyperinsulinemic-euglycemic clamps were performed with infusion of Intralipid (5 mL/ $/ \mathrm{kg} / \mathrm{h}) / \mathrm{heparin}(6 \mathrm{U} / \mathrm{hour})$ or heparin (6 U/hour) alone. GIR and GIR at steady state (bar graph) (C), EGP and percent suppression of EGP by insulin (D), and C-peptide levels (E) during clamps $\left(2.5 \mathrm{mU} / \mathrm{min} / \mathrm{kg}\right.$ insulin infusion rate) in vehicle- and PAHSA-treated (29 weeks) chow-fed mice. $n=6-8 /$ group. For C-E, ${ }^{*} P<0.05$ versus vehicle and vehicle + Intralipid, ${ }^{\#} P=0.09$ versus PAHSA group, ${ }^{\dagger} P<0.05$ versus all other groups except PAHSA + Intralipid, ${ }^{\ddagger} P<0.05$ versus basal. GIR and GIR at steady state (bar graph) (F), insulin at the end of clamp (G), and EGP and percent suppression of EGP by insulin (H) during clamps (4 $\mathrm{mU} / \mathrm{min} / \mathrm{kg}$ insulin infusion rate) in vehicle- and PAHSA-treated (19 weeks) HFD-fed mice. $n=4$ /group. Statistical significance for $\mathbf{C}$ and $\mathbf{F}$ was evaluated by 2 -way repeatedmeasures ANOVA; for all others, 2-way ANOVA with Tukey's post hoc test or unpaired 2-tailed Student's $t$ test. Data are mean \pm SEM. 
ed previously by the failure of Intralipid infusion to prevent insulin suppression of EGP in chow-fed mice (6). These data are consistent with the notion that FFAs play a greater role in regulating EGP as the severity of insulin resistance increases $(6,39)$. Several studies demonstrated a clear association between increased nonesterified fatty acid levels and hyperglycemia in T2D subjects (40-42), but whether this plays a causative role is not known.

Our data indicate that PAHSAs also have direct effects on hepatocytes through a cAMP-dependent pathway. PAHSAs reduce basal and glucagon-stimulated glucose output in a cell-autonomous manner. The effect on glucagon-stimulated EGP may involve a reduction in glucagon-stimulated cAMP generation in primary hepatocytes. In contrast, PAHSAs have no effects on 8-bromocAMP-stimulated EGP, suggesting that PAHSAs decrease EGP by acting upstream of PKA. This concept is supported by our in vivo findings that PAHSA infusion decreases phosphorylation of CREB, a key cAMP-responsive PKA substrate, and the expression of CREB target genes Pck1 and G6pc1 (43) in the liver. PAHSAs also reduce the activity of G6pase, the rate-controlling glucose output enzyme, which is sensitive to cAMP (29). These data demonstrate that the direct effect of PAHSAs to suppress EGP requires inhibition of cAMP signaling, resulting in decreased G6pase activity.

We next sought to understand the signaling upstream of PAHSAs' direct effects on hepatocytes. We have previously shown that GPCRs of the $G \alpha_{\mathrm{q}}$ subtype mediate other biological functions of PAHSAs $(13,18,20)$. In the current study, PTX, a $G \alpha_{\text {i }}$ inhibitor, abolished the effects of PAHSAs on EGP, indicating that activation of a $G \alpha_{i}$ protein-coupled receptor is required for EGP suppression in response to PAHSAs.

We sought to determine whether PAHSAs also promote insulin-stimulated glucose uptake into muscle in vivo. We found increased glucose uptake, which could be explained by enhanced AKT signaling, in glycolytic (type II) fibers and heart in PAHSAtreated HFD-fed mice using a 4-mU insulin clamp dose. A higher insulin infusion rate is often required in order to achieve an effect on muscle glucose uptake compared with EGP due to the fact that EGP and lipolysis are more sensitive to insulin $(35,44)$. The increased glucose uptake in glycolytic muscle in PAHSA-treated mice could contribute to the enhanced systemic insulin sensitivity, because glycolytic fibers are more abundant than oxidative fibers in mice (45). Moreover, an increase in glycolytic muscle mass can result in metabolic improvement in obese mice (46). In contrast, glycolytic muscle fiber mass positively correlates with insulin resistance and obesity in humans $(47,48)$, but whether this contributes to, or is a response to, the insulin-resistant state is not known.

PAHSAs' effect of increasing glucose uptake in heart might be beneficial, because heart insulin resistance plays an important role in the pathogenesis of heart failure in individuals with T2D (49). Thus, our data suggest that increased glucose uptake in glycolytic fibers and heart by PAHSA treatment could be therapeutically beneficial. In PAHSA-treated chow-fed mice, we performed only a 2.5-mU insulin clamp, and we did not observe increased glucose uptake in extrahepatic tissues. This is expected, as we used an insulin dose that is optimized to detect changes in EGP, which is more sensitive to insulin than muscle glucose uptake (35). A greater increase in insulinemia as achieved with a 4-mU clamp in the HFD-fed mice is needed to stimulate muscle glucose uptake (44).
Endogenous insulin secretion also contributes to suppression of EGP (4), but that is not the explanation for the effects of PAHSAs, because we found no differences in insulin and/or C-peptide levels between PAHSA- and vehicle-treated mice on either a chow or HFD in several experimental paradigms. During acute i.v. PAHSA infusion, we did not use any stimulus to increase ambient glucose levels, and we actually observed a modest decrease in glycemia over 3 hours (data not shown). We previously reported that PAHSAs augmented oral glucose-stimulated insulin secretion in aged chow-fed mice (18). But in this study, we increased glycemia with i.p. pyruvate injection. The i.p. injection would bypass first-phase insulin secretion (50), which most likely explains why we did not see augmentation by PAHSAs of insulin secretion in aged chow-fed mice. We have not previously observed an effect of PAHSAs on insulin secretion in HFD-fed mice $(13,18)$.

In summary, we have identified target organs and biological mechanisms for the beneficial effects of PAHSAs on insulin sensitivity. Taken together, our data show that PAHSAs have multiple metabolic effects. This could be explained by the fact that PAHSAs activate multiple GPCRs that are present in different cell types and tissues and that exert different biologic effects in these tissues. Overall, it appears that in aged chow-fed mice, the effects of PAHSAs on GLP-1 and insulin secretion, combined with augmentation of insulin action to suppress EGP, are important for the improvement in glucose homeostasis. In HFD-fed mice, the in vivo mechanisms for improved glucose homeostasis appear to result more from PAHSAs' augmentation of insulin suppression of EGP, which results at least in part from their suppression of lipolysis. The PAHSA-mediated increase in insulin-stimulated glucose uptake in glycolytic muscle may also play a role in the beneficial effects in HFD-fed mice. Our data also show that in isolated hepatocytes, PAHSAs not only inhibit basal EGP directly, but also suppress glucagon-stimulated EGP through a cAMP-dependent pathway involving $\mathrm{G} \alpha_{\mathrm{i}}$ protein-coupled receptors.

Some effects of PAHSAs may be translatable from mice to humans, since the same PAHSA isomers are found in both species, and levels of many of these isomers are reduced in serum and subcutaneous adipose tissue in both insulin-resistant humans and mice (13). For insulin secretion, PAHSAs augment it in chow-fed but not HFD-fed mice. But PAHSAs directly improve glucosestimulated insulin secretion (GSIS) in human islets from both normal and T2D individuals, and restore the impaired pulsatility of insulin secretion in islets from T2D individuals (21). Determining the translatability of the findings in this study awaits development of PAHSAs suitable for administration to humans. Understanding the range of biological functions of PAHSAs and the underlying mechanisms will provide clarity regarding their roles in healthy and disease states.

\section{Methods}

Animal care. All C57BL/6J male mice were purchased from the Jackson Laboratory and were singly housed in ventilated cages with ad libitum access to food and water. Mice were kept on a 12-hour light/12-hour dark schedule at $22-23^{\circ} \mathrm{C}$. Before handling, mice were allowed to acclimate for at least 1 week in the animal facility. For PAHSA i.v. infusion studies, 16-week-old mice were fed a chow or HFD for 8 weeks, after which insulin resistance was confirmed by an ITT. For PAHSA 
A

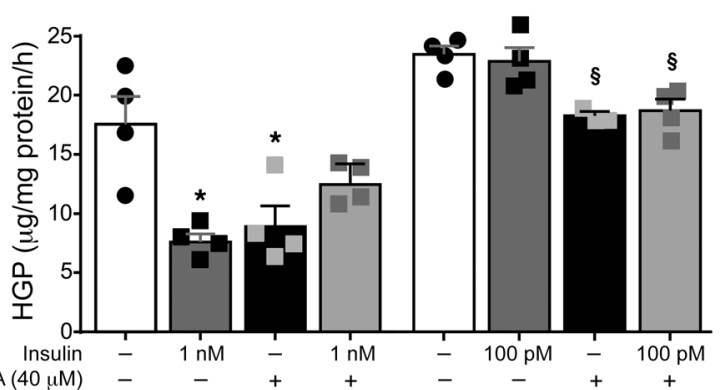

B

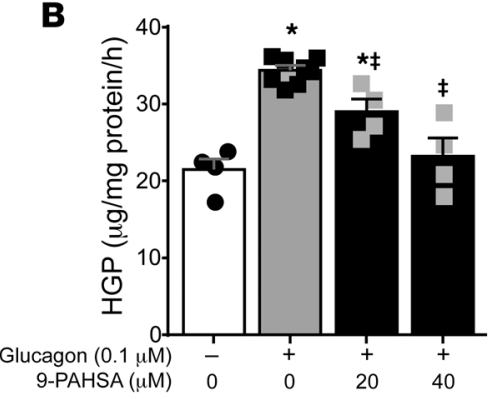

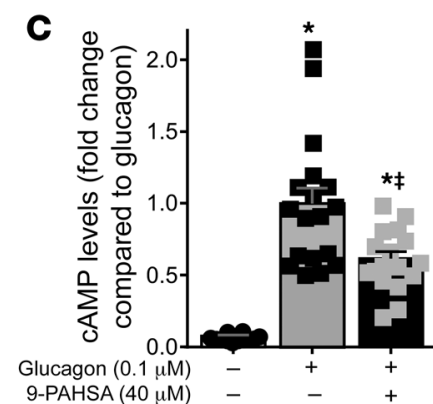
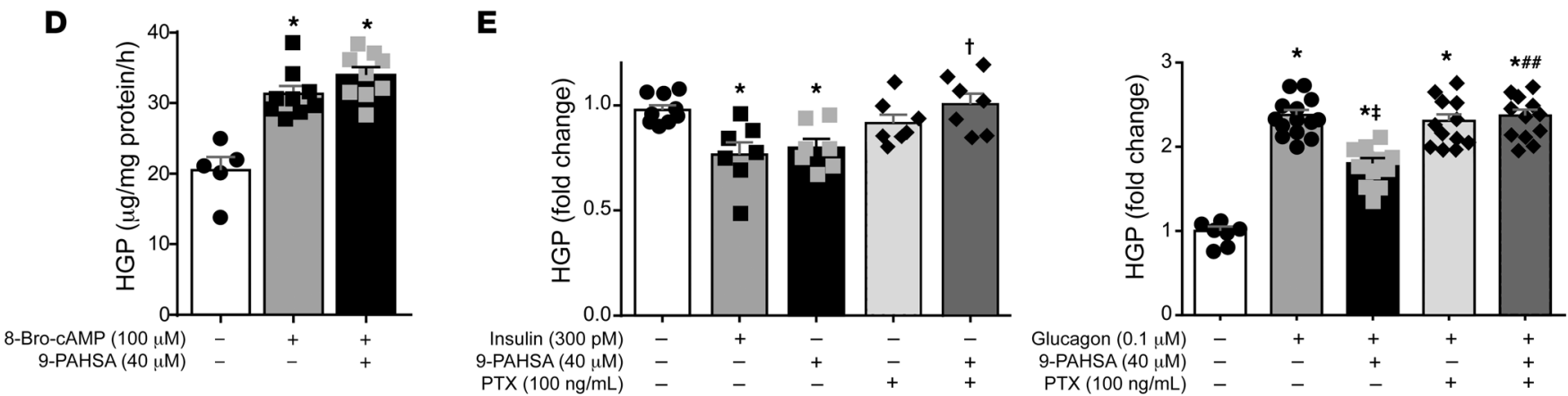

$\mathbf{F}$

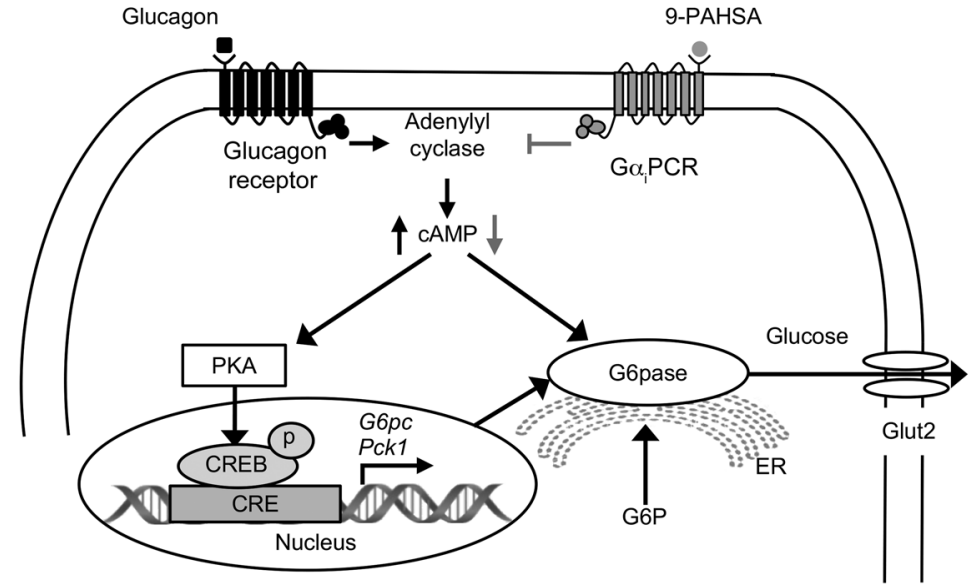

Figure 5. PAHSAs directly inhibit basal and glucagon-stimulated EGP in isolated hepatocytes through a cAMP-dependent pathway involving G $\alpha_{i}$ protein-coupled receptors. Hepatic glucose production (HGP) in murine primary hepatocytes treated with DMSO (0.2\%; white bars), 9-PAHSA, and/or insulin (A). $n=4$ wells/condition. HCP in hepatocytes treated with DMSO (0.2\%), glucagon, and 9-PAHSA + glucagon (B). $n=4-8$ wells/condition. (C) cAMP levels were measured 30 minutes after glucagon or 9-PAHSA + glucagon treatment. $n=12-18$ wells/condition. (D) HGP in primary hepatocytes treated with DMSO (0.2\%) (white bar), 8-bromo-cAMP (8-Bro-cAMP), or 9-PAHSA + 8-bromo-cAMP. $n=5$-9. (E) HCP in primary hepatocytes treated with DMSO (0.2\%) (white bars), insulin, 9-PAHSA, pertussis toxin (PTX), or 9-PAHSA + PTX (left); HGP in primary hepatocytes treated with DMSO (0.2\%), glucagon, 9-PAHSA, PTX, or glucagon + 9-PAHSA + PTX (right). $n=7-14$ wells/condition. For A-E, glucose output was measured 3 hours after treatment. ${ }^{*} P<0.05$ versus vehicle-treated control cells (first bar), ${ }^{\circledR} P<0.05$ versus vehicle- (fifth bar) or insulin-treated cells, ${ }^{\ddagger} P<0.05$ versus glucagon, ${ }^{\dagger} P<0.05$ versus 9 -PAHSA alone, and ${ }^{\#} P<0.05$ versus 9-PAHSA + glucagon. Statistical significance for all panels was evaluated by ANOVA followed by Tukey's post hoc tests or unpaired 2-tailed Student's $t$ test. Data are mean \pm SEM. (F) Model of the signaling pathway mediating PAHSAs' effects on HCP. In hepatocytes, glucagon increases EGP through the adenylyl cyclase/PKA/CREB pathway, whereas PAHSAs activate a $G \alpha_{i}$ GPCR (G $\left.\alpha_{i} P C R\right)$, inhibiting adenylyl cyclase, which decreases cAMP levels, reducing PKA activity. As a result, phosphorylation of CREB, a PKA protein target, is decreased, resulting in reduced expression of G6pc1 and Pck1, 2 CREB target genes. In addition, CAMP reduction by PAHSAs inhibits G6pase activity. The inhibition of these cAMP target proteins leads to reduced HGP by PAHSAs. Black arrows, effect of glucagon on PKA signaling pathway; gray arrows, effects of PAHSAs. CRE, cAMP response elements; G6P, glucose6-phosphate; Glut2, facilitated diffusion glucose transporter 2.

treatment studies, minipumps (Alzet, model 2006) filled with either vehicle (50\% polyethylene glycol 400 [PEG 400], 0.5\% Tween-80, $49.5 \%$ distilled water) or $5-(0.1 \mathrm{mg} / \mathrm{d})$ and 9-PAHSA $(0.4 \mathrm{mg} / \mathrm{d})$ were inserted subcutaneously into 12 -week-old mice, coinciding with the initiation of chow or HFD for up to 29 weeks. For catheter implanta- tion surgery, mice were anesthetized with an i.p. injection of ketamine (100 $\mathrm{mg} / \mathrm{kg}$ body weight) and xylazine $(10 \mathrm{mg} / \mathrm{kg}$ body weight), and an indwelling catheter was inserted in the right internal jugular vein. The catheters were externalized through an incision in the skin flap behind the head. No heparin was added in the catheters, except in the 
Intralipid infusion studies. After surgery, mice were kept on ad libitum chow or HFD for an additional 4 days. Body weight was monitored and catheters were flushed with $0.9 \%$ saline every day. For Intralipid infusion studies, catheters were flushed with $50 \mu \mathrm{L}$ heparinized saline every day before the clamp study. All mice used for the studies lost less than $10 \%$ of initial body weight. Glycemia was measured by tail bleeding using a glucometer (OneTouch). For all experiments, ageand body weight-matched animals were used.

Materials and chemical reagents. All chemical reagents were purchased from Sigma-Aldrich unless otherwise stated. p-CREB (catalog 9196), CREB (catalog 9197), p-AKT (catalog 4058), AKT (catalog 9272), p-GSK3 $\beta$ (catalog 5558), GSK3 $\beta$ (catalog 12456) and $\beta$-actin (catalog 3700) antibodies were purchased from Cell Signaling Technology. D- $\left[3-{ }^{3} \mathrm{H}\right]$-glucose and 2-DOG were purchased from PerkinElmer. INTRALIPID 20\% IV Fat Emulsion was purchased from Baxter. Heparin Sodium Injection was purchased from Pfizer Injectables. NEFA kits were obtained from Wako. Glucagon kits (Bio-Plex Pro Diabetes Assays) was obtained from Bio-Rad. ELISA kits were obtained from Crystal Chem (Mouse Insulin and C-Peptide), Abcam (Glycogen Assay Kit), and Cayman Chemical (Cyclic AMP ELISA Kit). Organic solvents for liquid chromatography-mass spectrometry (LC-MS) were purchased from Honeywell Burdick \& Jackson. Both 5- and 9-PAHSA were synthesized by Dionicio Siegel (Skaggs School of Pharmacy and Pharmaceutical Sciences, UCSD) based on established methods (51).

Measurement of metabolic parameters. For single PAHSA oral gavage studies, 17.5 hours after food removal ( 0.5 hours before initiation of the PTT) male mice were gavaged with $30 \mathrm{mg} / \mathrm{kg}$ body weight (chow-fed) 5- and 9-PAHSA in combination (50\% each) or an equivalent volume of vehicle (50\% PEG 400, 0.5\% Tween-80, 49.5\% distilled water). Mice received pyruvate ( $2 \mathrm{~g} / \mathrm{kg}$ body weight) by i.p. injection 30 minutes after PAHSA or vehicle administration. For PTT, glycemia was monitored every 15 minutes, mice were bled from the tail vein using non-heparin-coated capillary tubes, and serum insulin was measured by ELISA. For chronic PAHSA treatment studies, body weight and food intake were measured weekly, and body composition was measured by MRI. For glucagon measurement, mice were treated with vehicle or 5- and 9-PAHSAs for 5 weeks by subcutaneous minipumps. Five hours after food removal, serum was collected in tubes containing aprotinin (25 Kallikrein inhibitor units [KIU]/ $\mu \mathrm{L})$. Serum glucagon was then measured following the manufacturer's protocol. For pyruvate and lactate tolerance tests, 18-hour-fasted mice were injected with pyruvate and lactate $(1: 10,2 \mathrm{~g} / \mathrm{kg}$ body weight), and glycemia was monitored over a 2-hour period. For acute PAHSA infusion, after 5-hour food removal, $3{ }^{3} \mathrm{H}$ glucose $(0.05 \mu \mathrm{Ci} /$ $\mathrm{min})$ was infused through the jugular vein together with vehicle $(0.5 \%$ BSA and DMSO) or PAHSA (chow: $9 \mu \mathrm{g} /$ hour; HFD: $18 \mu \mathrm{g} /$ hour, preconjugated with $0.5 \% \mathrm{BSA}$ ) at rate of $3 \mu \mathrm{L} / \mathrm{min}$ for 3 hours. EGP was determined from the average for the time points over the last $45 \mathrm{~min}$ utes. The basal glucose clearance rate was calculated as the glucose disposal rate divided by the plasma glucose concentration, as previous described (52). For protein and RNA quantification, liver samples were freeze clamped, and other tissues were harvested and snap frozen in liquid nitrogen.

Hyperinsulinemic-euglycemic clamps. Hyperinsulemic-euglycemic clamps were performed in conscious and unrestrained 5-hour-fasted male mice, as previously described (35). In brief, $\left[3-{ }^{3} \mathrm{H}\right]$ glucose was continuously infused at the following rates: $t=-120$ to 0 minutes, 0.05 $\mu \mathrm{Ci} / \mathrm{min} ; t=0$ to 165 minutes, $0.1 \mu \mathrm{Ci} / \mathrm{min}$. The clamp was initiated at $t=0$ minutes with a continuous insulin infusion $(2.5 \mathrm{mU} / \mathrm{kg} / \mathrm{min}$ or $4 \mathrm{mU} / \mathrm{kg} / \mathrm{min}$ ). In the case of the $2.5-\mathrm{mU}$ infusion rate, at $t=0 \mathrm{a}$ bolus of insulin (16 mU/kg body weight) was given i.v. Glycemia was monitored every 10 minutes, and a variable amount of $30 \%$ glucose was administered to maintain euglycemia. EGP was determined from blood samples collected at -10 minutes and every 10 minutes from 125 to 165 minutes. Basal and insulin-stimulated EGP were determined by the $\left[3-{ }^{3} \mathrm{H}\right]$ glucose dilution method. 2 -DOG $(10 \mu \mathrm{Ci})$ was administered at 120 minutes to determine tissue-specific glucose uptake. For Intralipid infusion studies, prior to the initiation of the insulin portion of the clamp, a $100-\mu \mathrm{L}$ bolus of $10 \%$ Intralipid/heparin (3 U) was administered i.v. During the insulin clamp, Intralipid was infused at $5 \mathrm{~mL} / \mathrm{kg} / \mathrm{h}$ and heparin at $6 \mathrm{U} / \mathrm{h}$. At the end of the clamp, mice were sacrificed by ketamine injection. Liver samples were freeze clamped, and other tissues were removed following each experiment, snap frozen in liquid nitrogen, and stored at $-80^{\circ} \mathrm{C}$ for future experiments. Raw data for GIR and glycemia during the last 40 minutes (steady state) of Intralipid infusion are shown in Supplemental Table 1.

In vivo tissue glucose uptake and 2-DOG incorporation into liver glycogen. For 2.5-mU clamp studies, tissue samples were weighed and homogenized, and the supernatants were subjected to ion exchange column to separate 2-[ $\left[{ }^{14} \mathrm{C}\right] \mathrm{DOG}-6$-phosphate (2-DOGP) from 2-DOG. For the 4-mU clamp study, samples were weighed and homogenized in $0.5 \%$ perchloric acid. Homogenates were centrifuged and neutralized with $\mathrm{KOH}$. One aliquot was counted directly to determine 2-DOG and 2-DOGP radioactivity. A second aliquot was treated with $\mathrm{Ba}(\mathrm{OH})_{2}$ and $\mathrm{ZnSO}_{4}$ to remove 2-DOGP and then counted to determine 2-DOG. 2-DOG was calculated as the difference between the radioactivity of these 2 aliquots. In all experiments, the accumulation of 2-DOG was normalized to tissue weight.

2-DOG incorporation into glycogen was measured as previously described (33). In brief, liver samples (50-75 mg) were pulverized with mortar and pestle in liquid nitrogen, and digested by KOH. Once samples were dissolved, protein content was determined by the BCA assay. Glycogen was precipitated with $66 \%$ ethanol overnight. Glycogen concentration was determined by amyloglucosidase enzyme assays and corrected for sample protein content. To determine 2-DOG incorporation into liver glycogen, ${ }^{14} \mathrm{C}$ radioactivity in glycogen counts was divided by the integrated glucose-specific activity area under the curve and corrected for sample protein content.

Ex vivo lipolysis in adipose tissue explants. Lipolysis in adipose tissue explants was performed as previously described (19). In brief, perigonadal WAT was harvested from 20- to 33-week-old male chow-fed mice from the Jackson Laboratory and minced into 20- to 30-mg pieces. Explants were incubated in Krebs-Ringer-HEPES buffer (20 mM HEPES pH 7.4, $120 \mathrm{mM} \mathrm{NaCl}, 1.2 \mathrm{mM} \mathrm{CaCl}_{2}, 4.8 \mathrm{mM} \mathrm{KCl}, 0.6 \mathrm{mM} \mathrm{MgSO}_{4}$, $1.2 \mathrm{mM} \mathrm{KH}_{2} \mathrm{PO}_{4}$ ) with 3\% BSA, $200 \mathrm{nM}$ adenosine, and $5 \mathrm{mM}$ glucose with or without isoproterenol. The magnitude of the antilipolytic effect of 9-PAHSA was similar in the presence or absence of adenosine in the incubation media (Supplemental Figure 8). Insulin or 9-PAHSA was added 30 minutes before isoproterenol. Media were collected 2 hours after isoproterenol treatment, and FFA levels were determined using a colorimetric method according to the manufacturer's instructions (Wako NEFA kits). FFA levels were normalized to tissue weight.

PAHSA extraction and measurement. Lipid extraction was performed as previously described (13). Lipids were extracted from mouse 
serum (100 $\mu \mathrm{L}$, pooled from 3-4 mice). PBS was added to serum such that the final volume was $1 \mathrm{~mL}$. Subsequently, methanol and chloroform were added to this mixture (PBS/methanol/chloroform ratio was 1:1:2). The resulting mixture was shaken for 30 seconds by hand, vortexed for 15 seconds, and then centrifuged to separate the organic and aqueous phases. The organic phase containing lipids was then subjected to solid-phase extraction $(13,53)$.

G6pase activity assay. G6pase activity assay was performed as previously described (54). In brief, $100 \mathrm{mg}$ liver was lysed in buffer $(5 \mathrm{mM}$ HEPES; 250 mM sucrose) using a Dounce homogenizer. Microsomes were prepared using ultracentrifugation at $100,000 \mathrm{~g}$ and incubated for 20 minutes at $30^{\circ} \mathrm{C}$ with the substrate mix (G6P; sodium cacodylate; histone type IIA). The reaction was stopped with $4.5 \mathrm{mM}$ ammonium molybdate solution in $4 \%$ SDS. Absorbance was measured at $820 \mathrm{~nm}$.

Murine primary hepatocyte isolation and in vitro hepatic glucose production. Hepatocytes were isolated as previously described (55). Briefly, primary hepatocytes were isolated from 8- to 12-week-old male C57BL/6 mice from the Jackson Laboratory by perfusion with liver digest medium (Thermo Fisher Scientific), followed by $100-\mu \mathrm{m}$ cell strainer filtration. Viable hepatocytes were enriched by Percoll gradient centrifugation. The hepatocytes were resuspended in Williams' E Medium (Thermo Fisher Scientific) containing 10\% FBS and seeded on collagen-coated plates at a density of $0.25 \times 10^{6}$ cells/well (12-well plate). Four hours after attachment, cells were washed 3 times with phenol red-free DMEM with no glucose. Then, $300 \mu \mathrm{L}$ glucose production buffer (consisting of glucose-free DMEM pH 7.4 without phenol red, $20 \mathrm{mM}$ sodium lactate, and $2 \mathrm{mM}$ sodium pyruvate) containing PAHSAs, insulin, or glucagon was added to the cells. In all experiments, DMSO was used as vehicle at $0.2 \%$. Three hours later, media were collected, and glucose production was determined by the glucose assay kit and normalized to cellular protein concentrations. Before treatment, 9-PAHSA was maintained at $45^{\circ} \mathrm{C}$ and not mixed with glucagon or insulin. For glucagon treatment, cells were pretreated with 9-PAHSA for 30 minutes, and then glucagon was added in. Each experiment was repeated at least 3 times in 4-6 replicates.

Measurement of cellular cAMP levels. Thirty minutes after 9-PAHSA treatment, primary hepatocytes were incubated with glucagon for 30 minutes and immediately lysed with $110 \mu \mathrm{L}$ of $0.1 \mathrm{M} \mathrm{HCl}$. For measurement of cAMP levels, $250 \mu \mathrm{M}$ IBMX was included in all samples to inhibit cAMP hydrolysis. cAMP levels were then measured using a cAMP ELISA kit (Cayman Chemical) following the manufacturer's protocol for cell culture samples without acetylation.

Liver TG measurement. Liver TGs were extracted using the Folch method (56). A portion of the TG-containing chloroform phase was collected, evaporated, and resuspended in a 2:1 mixture of butanol and Triton X-100/methanol (3:1). TGs were then measured using a TG kit purchased from Stanbio according to the manufacturer's protocol and normalized to liver weight.

Western blotting. Cells and tissues were lysed with lysis buffer (50 mM HEPES, pH 7.5, 150 mM NaCl, 10\% glycerol, 1\% Triton X-100, 1.5 $\mathrm{mM} \mathrm{MgCl}$, $1 \mathrm{mM}$ EGTA, $10 \mathrm{mM}$ sodium pyrophosphate, $100 \mathrm{mM}$ sodi- um fluoride) containing a protease inhibitor cocktail. The lysates were sonicated and centrifuged at maximum speed for 15 minutes. Protein concentrations were determined using the BCA assay, and $60 \mu$ g protein was used for Western blotting. Protein extracts were resolved on SDSPAGE gel and transferred to nitrocellulose membranes (Bio-Rad). Proteins of interest were detected by incubation with secondary antibodies, followed by ECL detection reagent. Film images were scanned (Epson Expression $10000 \mathrm{XL}$ ) and quantified with Image software (NIH).

Gene expression. mRNA was extracted using TRIzol reagent (Invitrogen) and an RNeasy Mini kit (QIAGEN). cDNA was obtained using the Advantage RT-for-PCR kit (Clontech). Real-time PCRs for G6pc1 and Pck1 were performed on an ABI Prism 7900HT sequence detection system (Applied Biosystems) with TaqMan Universal PCR Master Mix and TaqMan gene expression assays (Applied Biosystems). Relative expression levels were determined using the $\Delta \Delta \mathrm{Ct}$ method. Target gene expression levels were normalized to GAPDH.

Statistics. All data are shown as mean \pm SEM. Statistical significance was determined by 2-tailed Student's $t$ test and/or 1- or 2-way ANOVA with Tukey's post hoc test for multiple comparisons. Significance was determined by repeated-measures ANOVA when required by the experimental design. Statistical analysis was performed with Prism 6.0 software (GraphPad Software). No data were excluded from the analyses. A $P$ value less than 0.05 was considered statistically significant.

Study approval. The present studies in animals were reviewed and approved by the Institutional Animal Care and Use Committee at Beth Israel Deaconess Medical Center.

\section{Author contributions}

PZ, A. Santoro, and ODP conceived of, designed, performed, and interpreted experiments and prepared figures. BBK conceived of, designed, and supervised the experimental plan and interpreted experiments. ATN, DS, and A. Saghatelian designed and performed chemical synthesis of the lipids. BBK, PZ, and A. Santoro wrote the manuscript. A. Saghatelian, DS, and ODP edited the manuscript. PZ initiated the project, and A. Santoro joined with critical skills and insights.

\section{Acknowledgments}

We thank Ismail Syed for technical assistance. This work was supported by NIH grants R01 DK43051 and P30 DK57521 (BBK) and R01 DK106210 (BBK and A. Saghatelian); a grant from the JPB Foundation (BBK); an American Heart Association (AHA) Postdoctoral Fellowship and American Diabetes Association grant 1-18-PDF-134 (PZ); and NIH grant F30 DK112622 (ATN).

Address correspondence to: Barbara B. Kahn, Division of Endocrinology, Diabetes and Metabolism, Department of Medicine, Beth Israel Deaconess Medical Center and Harvard Medical School, Center for Life Sciences, Room 747, 330 Brookline Ave, Boston, Massachusetts 02215, USA. Phone: 617.735.3324; Email: bkahn@ bidmc.harvard.edu.
1. Cho NH, et al. IDF Diabetes Atlas: global estimates of diabetes prevalence for 2017 and projections for 2045. Diabetes Res Clin Pract. 2018;138:271-281.

2. Wasserman DH. Four grams of glucose. Am J Physiol Endocrinol Metab. 2009;296(1):E11-E21.
3. Exton JH, Park CR. Control of gluconeogenesis in liver. II. Effects of glucagon, catecholamines, and adenosine 3',5'-monophosphate on gluconeogenesis in the perfused rat liver. J Biol Chem. 1968;243(16):4189-4196.
4. Edgerton DS, et al. Insulin's direct hepatic effect explains the inhibition of glucose production caused by insulin secretion. JCI Insight. 2017;2(6):e91863.

5. Perry RJ, et al. Hepatic acetyl CoA links adipose 
tissue inflammation to hepatic insulin resistance and type 2 diabetes. Cell. 2015;160(4):745-758.

6. Titchenell PM, et al. Direct hepatocyte insulin signaling is required for lipogenesis but is dispensable for the suppression of glucose production. Cell Metab. 2016;23(6):1154-1166.

7. Matsumoto M, Pocai A, Rossetti L, Depinho RA, Accili D. Impaired regulation of hepatic glucose production in mice lacking the forkhead transcription factor Foxo1 in liver. Cell Metab. 2007;6(3):208-216.

8. Cariou B, Charbonnel B, Staels B. Thiazolidinediones and PPAR $\gamma$ agonists: time for a reassessment. Trends Endocrinol Metab. 2012;23(5):205-215.

9. Chen Y, et al. Discovery of novel insulin sensitizers: promising approaches and targets. PPAR Res. 2017;2017:8360919.

10. Orgel E, Mittelman SD. The links between insulin resistance, diabetes, and cancer. Curr Diab Rep. 2013;13(2):213-222.

11. Ginsberg HN. Insulin resistance and cardiovascular disease. JClin Invest. 2000;106(4):453-458.

12. Biessels GJ, Despa F. Cognitive decline and dementia in diabetes mellitus: mechanisms and clinical implications. Nat Rev Endocrinol. 2018;14(10):591-604

13. Yore MM, et al. Discovery of a class of endogenous mammalian lipids with anti-diabetic and antiinflammatory effects. Cell. 2014;159(2):318-332.

14. Kuda O, et al. Docosahexaenoic acid-derived fatty acid esters of hydroxy fatty acids (FAHFAs) with anti-inflammatory properties. Diabetes. 2016;65(9):2580-2590.

15. Zhu QF, Yan JW, Zhang TY, Xiao HM, Feng YQ. Comprehensive screening and identification of fatty acid esters of hydroxy fatty acids in plant tissues by chemical isotope labeling-assisted liquid chromatography-mass spectrometry. Anal Chem. 2018;90(16):10056-10063.

16. Ma Y, Kind T, Vaniya A, Gennity I, Fahrmann JF, Fiehn O. An in silico MS/MS library for automatic annotation of novel FAHFA lipids. JCheminform. 2015;7:53.

17. Brezinova $\mathrm{M}$, et al. Levels of palmitic acid ester of hydroxystearic acid (PAHSA) are reduced in the breast milk of obese mothers. Biochim Biophys Acta Mol Cell Biol Lipids. 2018;1863(2):126-131.

18. Syed I, et al. Palmitic acid hydroxystearic acids activate GPR40, which is involved in their beneficial effects on glucose homeostasis. Cell Metab. 2018;27(2):419-427.e4.

19. Vijayakumar A, et al. Absence of carbohydrate response element binding protein in adipocytes causes systemic insulin resistance and impairs glucose transport. Cell Rep. 2017;21(4):1021-1035.

20. Lee J, et al. Branched fatty acid esters of hydroxy fatty acids (FAHFAs) protect against colitis by regulating gut innate and adaptive immune responses. J Biol Chem. 2016;291(42):22207-22217.

21. Bandak B, Yi L, Roper MG. Microfluidic-enabled quantitative measurements of insulin release dynamics from single islets of Langerhans in response to 5-palmitic acid hydroxy stearic acid. Lab Chip. 2018;18(18):2873-2882.

22. Hammarstedt A, et al. Adipose tissue dysfunction is associated with low levels of the novel palmitic acid hydroxystearic acids. Sci Rep. 2018;8(1):15757.

23. Wang YM, Liu HX, Fang NY. 9-PAHSA promotes browning of white fat via activating G-proteincoupled receptor 120 and inhibiting lipopolysaccharide / NF-kappa B pathway. Biochem Biophys Res Commun. 2018;506(1):153-160.

24. Pflimlin E, et al. Acute and repeated treatment with 5-PAHSA or 9-PAHSA isomers does not improve glucose control in mice. Cell Metab. 2018;28(2):217-227.e13.

25. Kuda O. On the complexity of PAHSA research. Cell Metab. 2018;28(4):541-542.

26. Syed I, et al. Methodological issues in studying PAHSA biology: masking PAHSA effects. Cell Metab. 2018;28(4):543-546.

27. Wang YM, Liu HX, Fang NY. High Glucose concentration impairs 5-PAHSA activity by inhibiting AMP-activated protein kinase activation and promoting nuclear factor-kappa-B-mediated inflammation. Front Pharmacol. 2018;9:1491.

28. Jiang G, Zhang BB. Glucagon and regulation of glucose metabolism. Am JPhysiol Endocrinol Metab. 2003;284(4):E671-E678.

29. Soty M, et al. Post-translational regulation of the glucose-6-phosphatase complex by cyclic adenosine monophosphate is a crucial determinant of endogenous glucose production and is controlled by the glucose-6-phosphate transporter. JProteome Res. 2016;15(4):1342-1349.

30. Burgess SC, et al. Cytosolic phosphoenolpyruvate carboxykinase does not solely control the rate of hepatic gluconeogenesis in the intact mouse liver. Cell Metab. 2007;5(4):313-320.

31. Zingone A, et al. Correction of glycogen storage disease type 1a in a mouse model by gene therapy. J Biol Chem. 2000;275(2):828-832.

32. Zheng D, Ionut V, Mooradian V, Stefanovski D, Bergman RN. Portal glucose infusion-glucose clamp measures hepatic influence on postprandial systemic glucose appearance as well as whole body glucose disposal. Am J Physiol Endocrinol Metab. 2010;298(2):E346-E353.

33. Zisman A, et al. Targeted disruption of the glucose transporter 4 selectively in muscle causes insulin resistance and glucose intolerance. $\mathrm{Nat}$ Med. 2000;6(8):924-928.

34. Parniak M, Kalant N. Incorporation of glucose into glycogen in primary cultures of rat hepatocytes. Can JBiochem Cell Biol. 1985;63(5):333-340.

35. Ayala JE, Bracy DP, McGuinness OP, Wasserman DH. Considerations in the design of hyperinsulinemic-euglycemic clamps in the conscious mouse. Diabetes. 2006;55(2):390-397.

36. Lu B, et al. Metabolic crosstalk: molecular links between glycogen and lipid metabolism in obesity. Diabetes. 2014;63(9):2935-2948.

37. Petersen MC, Vatner DF, Shulman GI. Regulation of hepatic glucose metabolism in health and disease. Nat Rev Endocrinol. 2017;13(10):572-587.

38. Lu M, et al. Insulin regulates liver metabolism in vivo in the absence of hepatic Akt and Foxo1. Nat Med. 2012;18(3):388-395.

39. Lam TK, Carpentier A, Lewis GF, van de Werve G, Fantus IG, Giacca A. Mechanisms of the free fatty acid-induced increase in hepatic glucose production. Am J Physiol Endocrinol Metab. 2003;284(5):E863-E873.

40. Golay A, Swislocki AL, Chen YD, Reaven GM. Relationships between plasma-free fatty acid concentration, endogenous glucose production, and fasting hyperglycemia in normal and noninsulin-dependent diabetic individuals. Metab Clin Exp. 1987;36(7):692-696.

41. Swislocki AL, Chen YD, Golay A, Chang MO, Reaven GM. Insulin suppression of plasma-free fatty acid concentration in normal individuals and patients with type 2 (non-insulin-dependent) diabetes. Diabetologia. 1987;30(8):622-626.

42. Paolisso G, Tataranni PA, Foley JE, Bogardus C, Howard BV, Ravussin E. A high concentration of fasting plasma non-esterified fatty acids is a risk factor for the development of NIDDM. Diabetologia. 1995;38(10):1213-1217.

43. Hanson RW, Reshef L. Regulation of phosphoenolpyruvate carboxykinase (GTP) gene expression. Annu Rev Biochem. 1997;66:581-611.

44. O'Doherty RM, Halseth AE, Granner DK, Bracy DP, Wasserman DH. Analysis of insulin-stimulated skeletal muscle glucose uptake in conscious rat using isotopic glucose analogs. Am JPhysiol. 1998;274(2):E287-E296.

45. Hesse B, Fischer MS, Schilling N. Distribution pattern of muscle fiber types in the perivertebral musculature of two different sized species of mice. Anat Rec (Hoboken). 2010;293(3):446-463.

46. Izumiya $Y$, et al. Fast/glycolytic muscle fiber growth reduces fat mass and improves metabolic parameters in obese mice. Cell Metab. 2008;7(2):159-172.

47. Nyholm B, et al. Evidence of an increased number of type IIb muscle fibers in insulin-resistant first-degree relatives of patients with NIDDM. Diabetes. 1997;46(11):1822-1828.

48. Lillioja S, et al. Skeletal muscle capillary density and fiber type are possible determinants of in vivo insulin resistance in man. JClin Invest. 1987;80(2):415-424.

49. Abdul-Ghani M, DeFronzo RA, Del Prato S, Chilton R, Singh R, Ryder REJ. Cardiovascular disease and type 2 diabetes: has the dawn of a new era arrived? Diabetes Care. 2017;40(7):813-820.

50. McGuinness OP, Ayala JE, Laughlin MR, Wasserman DH. NIH experiment in centralized mouse phenotyping: the Vanderbilt experience and recommendations for evaluating glucose homeostasis in the mouse. Am J Physiol Endocrinol Metab. 2009;297(4):E849-E855.

51. Nelson AT, et al. Stereochemistry of endogenous palmitic acid ester of 9-hydroxystearic acid and relevance of absolute configuration to regulation. JAm Chem Soc. 2017;139(13):4943-4947.

52. Scarlett JM, et al. Central injection of fibroblast growth factor 1 induces sustained remission of diabetic hyperglycemia in rodents. Nat Med. 2016;22(7):800-806

53. Zhang T, et al. A LC-MS-based workflow for measurement of branched fatty acid esters of hydroxy fatty acids. Nat Protoc. 2016;11(4):747-763.

54. Alegre M, Ciudad CJ, Fillat C, Guinovart JJ. Determination of glucose-6-phosphatase activity using the glucose dehydrogenase-coupled reaction. Anal Biochem. 1988;173(1):185-189.

55. Hong S, et al. Nicotinamide N-methyltransferase regulates hepatic nutrient metabolism through Sirt1 protein stabilization. Nat Med.2015;21(8):887-894.

56. Folch J, Lees M, Sloane Stanley GH. A simple method for the isolation and purification of total lipides from animal tissues. J Biol Chem. 1957;226(1):497-509. 